T. Fujiwara and H. Kunita

Nagoya Math. J.

Vol. 127 (1992), 83-116

\title{
LIMIT THEOREMS FOR STOCHASTIC DIFFERENCE-DIFFERENTIAL EQUATIONS
}

\section{TSUKASA FUJIWARA AND HIROSHI KUNITA}

\section{Introduction}

There are extensive works on the limit theorems for sequences of stochastic ordinary differential equations written in the form:

$$
\frac{d \varphi_{t}}{d t}=f_{t}^{n}\left(\varphi_{t}\right)+\bar{g}_{t}^{n}\left(\varphi_{t}\right)
$$

where $f_{t}^{n}=f_{t}^{n}(x), t \geq 0$ is a stochastic process and $\bar{g}_{t}^{n}=\bar{g}_{t}^{n}(x), t \geq 0$ is a deterministic function, both of which take values in the space of vector fields. The case where $\left\{f_{t}^{n}\right\}_{n}$ satisfies certain mixing conditions has been studied by Khas'minskii [7], Kesten-Papanicolaou [6] and others. The limit process is characterized as a diffusion process governed by a stochastic differential equation based on a Brownian motion. Further, the approximation theorem of stochastic differential equation studied by Wong-Zakai [18], Ikeda-Watanabe [4] etc. is also formulated in this way. A unified method of treating these problems was proposed by Kunita [9].

On the other hand, a lot of attention has also been shown to the discrete time approximation of stochastic differential equations. Approximating sequence of equations is written as

$$
\varphi_{k+1}=\varphi_{k}+f_{k}^{n}\left(\varphi_{k}\right) \xi_{k}^{n}+\bar{g}_{k}^{n}\left(\varphi_{k}\right), k=1,2, \ldots
$$

where $\left\{\xi_{k}^{n}\right\}$ is an array of random variables with certain mixing conditions and $\left\{f_{k}^{n}, \bar{g}_{k}^{n}\right\}_{n}$ is an array of continuous maps of the state space into itself. See Kushner [13], H. Watanabe [17] and Fujiwara [2]. The limit process is either a diffusion process mentioned above or a diffusion process with jumps governed by a stochastic differential equation based on a Lévy process.

In this paper we will present a unified method which is applicable both to stochastic ordinary differential equation (1.1) and to stochastic difference 
equation (1.2). Equations are written as stochastic difference-differential equations of the form:

$$
\varphi_{t}^{n}=x_{0}+\int_{0}^{t}\left\{f_{u}^{n}\left(\varphi_{u-}^{n}\right)+\bar{g}_{u}^{n}\left(\varphi_{u-}^{n}\right)\right\} d A_{u}^{n} .
$$

Here $\left\{f_{u}^{n}\right\}$ is a sequence of vector field valued stochastic processes and $\left\{\bar{g}_{u}^{n}\right\}$ is a sequence of deterministic vector field valued functions of $u$. $\left\{A_{u}^{n}\right\}$ is a sequence of deterministic nondecreasing càdlàg (right continuous with left hand limits) functions of $u$.

Assumptions required for equations (1.3) and techniques employed for the proof of the limit theorem, are closely related to those in [9], where stochastic ordinary differential equations are discussed. However there are some important differences. In this paper, we get a sharper estimate of the solution. This enabled us to obtain a better result than authors' previous works. Another different point in this paper is that we introduced Lévy measures so that the limit process can have jumps and can be characterized as a Markov process associated with Lévy's infinitesimal generator.

The organization of this paper is as follows. In the next section we state our main theorem (Theorem 2.1). Assumptions on equations are presented in an abstract manner. They appear to be technical and complicated. A reason why we presented the theorem in this form is that we want to apply the theorem to various types of limit theorems. The theorem will be proved at Section 3. Since the proof is long, it will be divided into four subsections.

In the last section we will apply the theorem to stochastic ordinary differential equations. Two cases will be discussed separately. The first is the case where certain uniform mixing (or $\phi$-mixing) conditions are satisfied (Theorem 4.2). The second is the case where certain strong mixing conditions are satisfied (Theorem 4.6). Our typical results are Corollaries 4.5 and 4.8. There we discuss the weak convergence of solutions of equations

$$
\frac{d \varphi_{t}}{d t}=\sqrt{n} f\left(\varphi_{t}, n t\right)
$$

where $f(x, t)=\left(f^{1}(x, t), \ldots, f^{d}(x, t)\right)$ is a stationary process with mean 0 . We show that the sequence of solutions $\left\{\varphi^{n}\right\}$ converges in law to a diffusion process if the following $(a)$ and $(b)$ are satisfied:

(a): $E\left[\sup _{|x| \leq N}\left|\partial_{x}^{\alpha} f(x, 0)\right|^{2}\right]<\infty$ for all $N>0$ and $|\alpha| \leq 2$.

(b): The uniform mixing rate $\phi(t)$ satisfies $\int_{0}^{\infty} \phi(s)^{1 / 2} d s<\infty$,

or if the following $(a)_{\delta}^{\prime}$ and $(b)_{\gamma}^{\prime}$ are satisfied for some $\delta \in(0, \infty)$ and $\gamma \in$ $(0, \delta / 2(2+\delta)(1+d))$. 
$(a)_{\delta}^{\prime}: E\left[\sup _{|x| \leq N}\left|\partial_{x}^{\alpha} f(x, 0)\right|^{2+\delta}\right]<\infty$ for all $N>0$ and $|\alpha| \leq 2$,

$(b)_{\gamma}^{\prime}$ : The strong mixing rate $\alpha(t)$ satisfies $\int_{0}^{\infty} \alpha(s)^{\gamma} d s<\infty$.

Here $\partial_{x}^{\alpha}=\left(\partial / \partial x^{1}\right)^{\alpha_{1}} \cdots\left(\partial / \partial x^{d}\right)^{\alpha_{d}}$ and $|\alpha|=\alpha_{1}+\cdots+\alpha_{d}$. The diffusion and drift coefficients (characteristics) of the limit process are given by

$$
\begin{aligned}
& a^{i j}(x)=E\left[\left(\int_{0}^{\infty} f^{i}(x, s) d s\right) f^{j}(x, 0)\right]+E\left[\left(\int_{0}^{\infty} f^{j}(x, s) d s\right) f^{i}(x, 0)\right], \\
& b^{i}(x)=\sum_{j=1}^{d} E\left[\left(\int_{0}^{\infty} \frac{\partial f^{i}}{\partial x^{j}}(x, s) d s\right) f^{j}(x, 0)\right] .
\end{aligned}
$$

Similar limit theorems have been discussed by many authors. See Khas'minskii [7], Kesten-Papanicolaou [6], Kunita [8], [9], Kushner [13], and references therein. Conditions assumed in these works are much stronger than ours. Concerning moment conditions, $f(x, t)$ is assumed to be bounded in [7], [13] and is assumed to satisfy Condition $(a)_{\delta}^{\prime}$ with $\delta>2$ in [9]. It seems to us that our present condition ( $a$ ) (existence of the second moment) would be the best possible one as far as the moment conditions are concerned, since our assertion can be regarded as a central limit theorem. Conditions $(a)_{\delta}^{\prime}$ and $(b)_{\gamma}^{\prime}$ with $\delta \in(0, \infty), \gamma \in$ $(0, \delta / 2(2+\delta)(1+d))$ is also a relaxization of conditions in [6] and [8].

Another interesting application of our main theorem will be to the sequence of stochastic difference equations (1.2). We show in Fujiwara [3] that the sequence of solutions of stochastic difference equations of the form (1.2) converges in law to a certain Markov process with jumps under mixing conditions for $\left\{\xi_{k}^{n}\right\}$ similar to $(a)$ or $(a)_{\delta}^{\prime}$. Futher, we show in Kunita [11] that the sequence of random measures of the form

$$
B^{n}(t, E)=\frac{1}{\sqrt{n}} \sum_{k=1}^{[n t]}\left\{I_{E}\left(\sqrt{n} \xi_{k}^{n}\right)-\pi_{n}(E)\right\},
$$

where $I_{E}$ is the indicator function of the set $E$ and $\pi_{n}$ is the law of $\sqrt{n} \xi_{1}^{n}$, converges in law to a Brownian random measure $B(t, E)$ and the sequence of random measures

$$
N^{n}(t, F)=\sum_{k=1}^{[n t]} I_{F}\left(\xi_{k}^{n}\right),
$$

converges to a Poisson random measure $N(t, F)$. These limit theorems are applied for the limit theorem of solutions of stochastic difference equations (1.2).

\section{A convergence theorem}

We begin by introducing some function spaces. Let $k$ be a nonnegative integer 
and let $\mathbf{C}^{k}=\mathbf{C}^{k}\left(\mathbf{R}^{d}, \mathbf{R}^{d}\right)$ be the set of all $\mathbf{C}^{k}$-maps from $\mathbf{R}^{d}$ into itself. For $f \in$ $\mathbf{C}^{k}$, we define the norm $\|f\|_{k}^{*}$ by

$$
\|f\|_{k}^{*}=\sup _{x \in \mathbf{R}^{d}}\left(\frac{|f(x)|}{1+|x|}\right)+\sum_{1 \leq|\alpha| \leq k} \sup _{x \in \mathbf{R}^{d}}\left|\partial_{x}^{\alpha} f(x)\right| .
$$

We denote by $\mathbf{C}_{b *}^{k}$ the set of all $f \in \mathbf{C}^{k}$ such that $\|f\|_{k}^{*}<\infty$. The space $\mathbf{C}_{b *}^{k}$ and the norm $\|f\|_{0}^{*}$ are often denoted by $\mathbf{C}_{b *}$ and $\|f\|^{*}$. We also denote by $\|f\|\left(\|f\|_{k}\right)$ the supremum norm (the $\mathbf{C}^{k}$-norm, respectively).

Now let $(\Omega, \mathscr{F}, P)$ be a probability space with a family of filtrations $\left\{\mathscr{F}_{t}^{n}\right.$; $t \geq 0\}_{n \in \mathbf{N}}$. Suppose that for each $n \in \mathbf{N}$ we are given an $\left\{\mathscr{F}_{t}^{n}\right\}_{\text {-adapted càdlàg }}$ stochastic process $f_{t}^{n}=f_{t}^{n}(x), t \geq 0$ with values in $\mathbf{C}_{b *}^{2}$ and a determinitic càdlàg function $\bar{g}_{t}^{n}=\bar{g}_{t}^{n}(x), t \geq 0$ with values in $\mathbf{C}_{b *}^{1}$. Suppose further that we are given a sequence of nondecreasing, deterministic and càdlàg functions $\left\{A_{t}^{n}\right\}$ such that $A_{0}^{n}=0$. We often use the following abbreviations. For nondecreasing processes $A$ and $B$, we denote by $A \ll B$ if $B-A$ is also nondecreasing. We set $\Delta A_{t}^{n}=A_{t}^{n}-A_{t-}^{n}$,

$$
f_{u, \delta}^{n}(x)=f_{u}^{n}(x) I_{\left\{\left\|f_{u}^{n} \Delta A_{\|}^{n}\right\|^{*} \leq \delta\right\}}, \quad f_{u, M}^{n, \delta}(x)=f_{u}^{n}(x) I_{\left\{\delta<\left\|f_{u}^{n} \Delta A_{u}^{n}\right\|_{*}^{*} \leq M\right)},
$$

and

$$
\bar{f}_{u, \delta}^{n}(x)=E\left[f_{u, \delta}^{n}(x)\right], \quad \tilde{f}_{u, \delta}^{n}(x)=f_{u, \delta}^{n}(x)-\bar{f}_{u, \delta}^{n}(x),
$$

for $0<\delta \leq M$, where it is assumed that the expectations $\bar{f}_{u, \delta}^{n}(x)$ exist for all

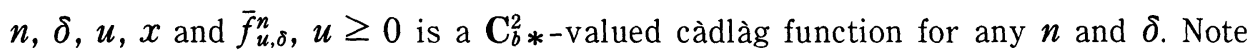
that $f_{u, \delta}^{n}=f_{u}^{n}$ and $f_{u, M}^{n, \delta}=0$ hold if $\Delta A_{u}^{n}=0$. We denote by $f_{u}^{n,(i)}$ the $i$-th component of $f_{u}^{n}$. We also denote by $\mathbf{R}^{d} \otimes \mathbf{R}^{e}\left(\mathbf{S}_{d}\right)$ the set of all $d \times e$ real matrices (the set of all $d \times d$ real, symmetric, nonnegative definite matrices, respectively).

Consider the sequence of stochastic difference-differential equations (1.3). For almost all $\omega \in \Omega$, it has a unique global solution $\varphi_{t}^{n}, t \geq 0$. It is a càdlàg process with values in $\mathbf{R}^{d}$. The purpose of this paper is to discuss the weak convergence of the sequence of càdlàg processes $\left\{\varphi_{t}^{n}\right\}_{n}$. Let $\mathbf{D}_{d}=\mathbf{D}\left([0, \infty), \mathbf{R}^{d}\right)$ be the space of all càdlàg functions from $[0, \infty)$ to $\mathbf{R}^{d}$, endowed with Skorohod's $J_{1}$-topology. See Jacod-Shiryaev [5] for the Skorohod space. We denote by $\mathscr{B}\left(\mathbf{D}_{d}\right)$ its topological Borel field. Then the law of the càdlàg process $\varphi_{t}^{n}, t \geq 0$ can be defined on the space $\left(\mathbf{D}_{d}, \mathscr{B}\left(\mathbf{D}_{d}\right)\right)$ as usual. The sequence of càdlàg processes $\left\{\varphi_{t}^{n}\right\}_{n}$ is said to converge in law if the corresponding sequence of laws on $\left(\mathbf{D}_{d}, \mathscr{B}\left(\mathbf{D}_{d}\right)\right)$ converges weakly.

To establish the weak convergence of $\left\{\varphi^{n}\right\}_{n}$ we introduce the following system of conditions (A. I ) (A.I) for $\left\{f_{t}^{n}(x), \bar{g}_{t}^{n}(x), A_{t}^{n}\right\}_{n}$. 
(A.I): (1) For every compact set $K$ in $\mathbf{R}^{d}$ and positive constants $M, T$, there exists a sequence of nondecreasing càdlàg processes $\left\{D^{n}\right\}_{n}$ satisfying following properties (i) and either, (ii) or (ii) ${ }^{\prime}$

(i) For all $s \leq t \leq t^{\prime} \leq T$,

$$
\begin{aligned}
\sum_{|\alpha| \leq 2,|\beta| \leq 1}\left\{\int_{(s, t]} \sup _{x, y \in K} \mid\right. & E\left[\int_{\left(v, t^{\prime}\right]} \partial_{y}^{\alpha} \tilde{f}_{u, M}^{n}(y) d A_{u}^{n} \mid \mathscr{F}_{v}^{n}\right]|| \partial_{x}^{\beta} \tilde{f}_{v, M}^{n}(x) \mid d A_{v}^{n} \\
& \left.+\int_{(s, t]} \sup _{x \in K}\left|\partial_{x}^{\beta} f_{v, M}^{n}(x)\right|^{2} \Delta A_{v}^{n} d A_{v}^{n}\right\} \leq D_{t}^{n}-D_{s}^{n} .
\end{aligned}
$$

(ii) The sequence of compensators $\left\{D^{n, p}\right\}_{n}$ of $\left\{D^{n}\right\}_{n}$ is $C$-tight in the space $\mathbf{D}_{1}$, that is, it is tight and any limit law is supported on the space of continuous paths $\mathbf{C}\left([0, \infty), \mathbf{R}^{1}\right)$. In addition, $\left\{D_{t}^{n, p}\right\}_{n}$ is uniformly integrable for each $t$.

(ii) ${ }^{\prime}\left\{D^{n}\right\}_{n}$ is $C$-tight in the space $\mathbf{D}_{1}$ and $\left\{D_{t}^{n}\right\}_{n}$ is uniformly integrable for each $t$.

(2) For every compact set $K$ and for every pair of positive constants $\delta<M$, there exists a sequence of deterministic nondecreasing functions $\left\{\bar{D}^{n}\right\}_{n}$ satisfying the following properties.

(i)

$$
\begin{aligned}
\sum_{|\beta| \leq 1}\left\{\int_{(0, t]} \sup _{x \in K}\left|\partial_{x}^{\beta}\left(\bar{f}_{u, M}^{n}(x)+\bar{g}_{u}^{n}(x)\right)\right| d A_{u}^{n}\right. \\
\left.\quad+\int_{(0, t]} E\left[\sup _{x \in K}\left|\partial_{x}^{\beta} f_{u, M}^{n, \delta}(x)\right|\right] d A_{u}^{n}\right\} \ll \bar{D}_{t}^{n} .
\end{aligned}
$$

(ii) For every $T>0, \sup _{n \in \mathbf{N}} \bar{D}_{T}^{n}$ and

$$
\lim _{\theta \downarrow 0} \limsup _{n \rightarrow \infty} \sup _{s<t \leq T, t-s \leq \theta}\left(\bar{D}_{t}^{n}-\bar{D}_{s}^{n}\right)=0 .
$$

(A.II): (1) There exists a family of $\sigma$-finite measures $\nu_{u}(d f), u \geq 0$ on $\mathbf{C}_{b *}$ satisfying the following properties of (i) and (ii).

(i) For every $T>0$

$$
\int_{0}^{T} \int_{\left\{\|f\|^{*} \leq 1\right\}}\|f\|_{1}^{* 2} \nu_{u}(d f) d u<\infty \text { and } \int_{0}^{T} \nu_{u}\left(\|f\|^{*}>1\right) d u<\infty .
$$

(ii) For every bounded continuous function $h$ on $\mathbf{R}^{d}$, compact set $K$ in $\mathbf{R}^{d}$, and $\delta<M$ such that $\delta, M \in C(\nu)=:\left\{r>0 ; \int_{0}^{T} \nu_{u}(\{f ;\|f\| *=r\}) d u=0\right.$ for any $T>0\}$,

$$
\begin{aligned}
& \lim _{n \rightarrow \infty} E\left[\sup _{x \in K} \mid E\left[\sum_{s<u \leq t} h\left(f_{u}^{n}(x) \Delta A_{u}^{n}\right) I_{\left\{\delta<\left\|f_{u}^{n} \Delta A_{s}^{n}\right\|^{*} \leq M\right)} \mid \mathscr{F}_{s}^{n}\right]\right. \\
&\left.-\int_{s}^{t} \int_{C_{b *}} h(f(x)) I_{\left\{\delta<\|f\|^{*} \leq M\right)} \nu_{u}(d f) d u \mid\right]=0 .
\end{aligned}
$$


In addition, $1 \in C(\nu)$.

(2) There exists a càdlàg function $a_{0}(u)=a_{0}(x, u)$ with values in $\mathbf{C}_{b *}^{2}\left(\mathbf{R}^{d}, \mathbf{S}_{d}\right)$ such that for every compact set $K$ and $s \leq t$

$$
\begin{array}{r}
\lim _{\delta \in C(\nu) \downarrow 0} \limsup _{n \rightarrow \infty} E\left[\sup _{x \in K} \mid E\left[\int_{(s, t]} \tilde{f}_{u, \delta}^{n,(i)}(x) \tilde{f}_{u, \delta}^{n,(j)}(x) \Delta A_{u}^{n} d A_{u}^{n} \mid \mathscr{F}_{s}^{n}\right]\right. \\
\left.-\int_{s}^{t} a_{0}^{i j}(x, u) d u \mid\right]=0 .
\end{array}
$$

(3) There exist càdlàg functions $a_{1}(u)=a_{1}(x, u)$ with values in $\mathbf{C}_{b *}^{2}\left(\mathbf{R}^{d}, \mathbf{R}^{d} \otimes \mathbf{R}^{d}\right)$ and $c(u)=c(x, u)$ with values in $\mathbf{C}_{b *}^{1}\left(\mathbf{R}^{d}, \mathbf{R}^{d}\right)$ such that for every compact set $K, M>0$, and $s \leq t$

$$
\begin{aligned}
\lim _{n \rightarrow \infty} E\left[\sup _{x \in K} \mid E\left[\int_{(s, t]} \int_{(v, t]} \tilde{f}_{u, M}^{n,(i)}(x) \tilde{f}_{v, M}^{n,(j)}(x) d A_{u}^{n} d A_{v}^{n} \mid \mathscr{F}_{s}^{n}\right]\right. & \\
& \left.-\int_{s}^{t} a_{1}^{i j}(x, u) d u \mid\right]=0,
\end{aligned}
$$

and

(2.11) $\lim _{n \rightarrow \infty} E\left[\sup _{x \in K} \mid E\left[\int_{(s, t]} \int_{(v, t]} \sum_{j=1}^{d}\left(\frac{\partial}{\partial x^{j}} \tilde{f}_{u, M}^{n,(i)}\right)(x) \tilde{f}_{v, M}^{n,(j)}(x) d A_{u}^{n} d A_{v}^{n} \mid \mathscr{F}_{s}^{n}\right]\right.$

$$
\left.-\int_{s}^{t} c^{i}(x, u) d u \mid\right]=0
$$

respectively.

(4) There exists a càdlàg function $b(u)=b(x, u)$ with values in $\mathbf{C}_{b *}^{1}\left(\mathbf{R}^{d}, \mathbf{R}^{d}\right)$ such that for every compact set $K$ and $s \leq t$

$$
\left.\lim _{n \rightarrow \infty} \sup _{x \in K}\left|\int_{(s, t]}\left\{\bar{f}_{u, 1}^{n}(x)+\bar{g}_{u}^{n}(x)\right\} d A_{u}^{n}-\int_{s}^{t} b(x, u) d u\right|\right]=0 .
$$

(A.III): (1) For every compact set $K, s \leq t, M>0$,

$$
\lim _{n \rightarrow \infty} \sup _{s \in[0, t]} \sum_{|\alpha| \leq 2} E\left[\sup _{x \in K}\left|E\left[\int_{(s, t]} \partial_{x}^{\alpha} \tilde{f}_{u, M}^{n}(x) d A_{u}^{n} \mid \mathscr{F}_{s}^{n}\right]\right|\right]=0 .
$$

(2) For every compact set $K, s \leq t, M>0$,

$$
\begin{gathered}
\lim _{n \rightarrow \infty} \sum_{|\alpha| \leq 2} E\left[\int_{(s, t]} \sup _{x, y \in K}\left|E\left[\int_{(v, t]} \partial_{y}^{\alpha} \tilde{f}_{u, M}^{n}(y) d A_{u}^{n} \mid \mathscr{F}_{v}^{n}\right]\right|\right. \\
\left.\times\left|\tilde{f}_{v, M}^{n}(x)\right|^{2} \Delta A_{v}^{n} d A_{v}^{n}\right]=0 .
\end{gathered}
$$

(A.IV): For every $t>0$,

$$
\lim _{M \rightarrow \infty} \limsup _{n \rightarrow \infty} P\left[\sup _{u \leq t}\left\|f_{u}^{n} \Delta A_{u}^{n}\right\| *>M\right]=0 .
$$

Our main theorem in this paper is stated as follows. 
THEOREM 2.1. Assume (A.I) (A.IV). Then, the family of $\mathbf{D}_{d}$-valued random variables $\left\{\varphi^{n}\right\}_{n}$ satisfying (1.3) converges in law to the unique solution of the following stochastic differential equation:

$$
\begin{aligned}
\varphi_{t}= & x_{0}+\int_{(0, t]} \sigma\left(\varphi_{u-}, u\right) d B_{u}+\int_{(0, t]}(b+c)\left(\varphi_{u-}, u\right) d u \\
& +\int_{(0, t]} \int_{\left\{\|f\|^{*} \leq 1\right\}} f\left(\varphi_{u-}\right) \tilde{N}(d u d f) \\
& +\int_{(0, t]} \int_{\left\{\|f\|^{*}>1\right\}} f\left(\varphi_{u-}\right) N(\text { dudf }),
\end{aligned}
$$

where (i) $\sigma(u)=\sigma(x, u)$ is a càdlàg function with values in $\mathbf{C}_{b *}^{1}\left(\mathbf{R}^{d}, \mathbf{R}^{d} \otimes \mathbf{R}^{r}\right)$ such that $\sigma(u) \sigma(u)^{*}=a_{0}(u)+\left\{a_{1}(u)+a_{1}(u)^{*}\right\}$ where $a^{*}$ denotes the transpose of the matrix $a$,

(ii) $B_{t}$ is an $r$-dimensional standard Brounian motion, and

(iii) $N(d u d f)$ is a Poisson random measure with the intensity measure $d u \nu_{u}(d f)$ and $\tilde{N}(d u d f)$ denotes the compensated measure defined by $\tilde{N}(d u d f)=N(d u d f)-$ $d u \nu_{u}(d f)$.

See Ikeda-Watanabe [4] for stochastic integrals based on Poisson random measures and stochastic differential equations of jump type such as (2.16).

We defer the proof of Theorem 2.1 to the next section.

\section{Proof of Theorem 2.1}

In this section, we will give a proof of Theorem 2.1, by applying the so-called martingale method. However, since it seems diffcult to apply the method for $\left\{\varphi^{n}\right\}_{n}$ satisfying (1.3) itself, we divide the proof into several steps. First, we introduce a family of stochastic processes which are uniformly bounded and have uniformly bounded jumps, as follows. For given $L>0$, let $r_{L}(x)$ be a nonnegative smooth function from $\mathbf{R}^{d}$ to $\mathbf{R}^{1}$ such that $r_{L}(x) \equiv 1$ for $|x| \leq L$ and that $r_{L}(x) \equiv 0$ for $|x| \geq L+1$. We fix such a function $r_{L}$ for each $L$ in the following discussion. For given $M \in C(\nu)$ and $L>0$, define the localized and truncated process $\varphi^{n, M, L}$ of $\varphi^{n}$ as the solution of the following equation:

$$
\varphi_{t}^{n, M, L}=x_{0}+\int_{(0, t]}\left\{f_{u, M, L}^{n}\left(\varphi_{u-}^{n, M, L}\right)+\bar{g}_{u, L}^{n}\left(\varphi_{u-}^{n, M, L}\right)\right\} d A_{u}^{n},
$$

where we set $f_{u, M, L}^{n}(x)=r_{L}(x) f_{u, M}^{n}(x), \bar{g}_{u, L}^{n}(x)=r_{L}(x) \bar{g}_{u}^{n}(x)$. Then for arbitrary $T>0$ there exists a positive constant $C_{M, L, T}$ such that $\left|\varphi_{t}^{n, M, L}\right| \leq C_{M, L, T}$ and $\left|\Delta \varphi_{t}^{n, M, L}\right| \leq C_{M, L, T}$ for all $n, t \leq T$, and $\omega \in \Omega$. Throughout section 3 , we denote 
by $K$ the compact set $\left\{x ;|x| \leq C_{M, L, T}\right\}$.

The first step of our proof is to show the weak convergence of $\left\{\varphi^{n, M, L}\right\}_{n}$ for fixed $M$ and $L$. For this aim, we will apply the martingale method for $\left\{\varphi^{n, M, L}\right\}_{n}$. In fact, in subsection $3-1$, the tightness is shown. See Proposition 3.2. Further, in section $3-2$, it is shown that any weak limit is unique and coincides with the law of some stochastic differential equation. See Proposition 3.4. After establishing the first step (Proposition 3.10), we will proceed into the second step of removing the restriction of localization in (3.1). This problem is discussed in section 3-3. See Proposition 3.11. In final section 3-4, we will remove the restriction of truncation on jumps, and then we will complete our proof of Theorem 2.1. Though the outline of the proof follows that given in Fujiwara [2], it should be noted that the proof given below does not depend on the specific property of mixing arrays of random variables.

First of all we give a formula of the change of variables for the process $\varphi_{t}^{n}$, which will be often used in the proof of Theorem 2.1.

Lemma 3.1. Let $F(x), x \in \mathbf{R}^{d}$ be a $\mathbf{C}^{1}$-function and let $\phi_{t}=\left(\phi_{t}^{1}, \ldots, \phi_{t}^{d}\right)$ be an $\mathbf{R}^{d}$-valued càdlàg process of bounded variation. Set $\Delta \phi_{u}^{i}=\phi_{u}^{i}-\phi_{u-}^{i}$. Then for any $s<t$,

$$
\begin{aligned}
& F\left(\phi_{t}\right)-F\left(\phi_{s}\right) \\
& =\sum_{i=1}^{d} \int_{(s, t]} \frac{\partial F}{\partial x^{i}}\left(\phi_{u-}\right) d \phi_{u}^{i}+\sum_{s<u \leq t}\left(F\left(\phi_{u}\right)-F\left(\phi_{u-}\right)-\sum_{i=1}^{d} \frac{\partial F}{\partial x^{i}}\left(\phi_{u-}\right) \Delta \phi_{u}^{i}\right) \\
& =\sum_{i=1}^{d} \int_{(s, t]}\left\{\int_{0}^{1} \frac{\partial F}{\partial x^{i}}\left(\phi_{u-}+\theta \Delta \phi_{u}\right) d \theta\right\} d \phi_{u}^{i} .
\end{aligned}
$$

Here we note that

$$
\int_{0}^{1} \frac{\partial F}{\partial x^{i}}\left(\phi_{u-}+\theta \Delta \phi_{u}\right) d \theta=\frac{\partial F}{\partial x^{i}}\left(\phi_{u-}\right) \text { if } \Delta \phi_{u}=0 .
$$

The proof is easy and is left to the reader.

\section{3-1. Tightness of $\left\{\varphi^{n, M, L}\right\}_{n}$}

The aim of this subsection is to prove the following proposition.

Proposition 3.2. Let $M$ and $L$ be fixed. Then $\left\{\varphi^{n, M, L}\right\}_{n}$ of $(3.1)$ is tight in $\mathbf{D}_{d}$.

For simplicity, we discuss only on 1-dimensional case $(d=1)$. Also, in order to avoid the notational complexity, we consider the equation (3.1) as if $r_{L} \equiv 1$, and 
we omit the superscripts and the subscripts $M, L$ in $\varphi^{n, M, L}, f_{u, M}^{n}, \bar{f}_{u, M}^{n}, \tilde{f}_{u, M}^{n}, f_{u, M}^{n, \delta}$, and $\bar{f}_{u, M}^{n, \delta}$. Further, we will give the proof under the assumption (A.I)-(1)-(ii) because the proof goes similarly when we assume (A.I)-(1)-(ii)'. In order to show the tightness of $\left\{\varphi^{n}=\varphi^{n, M, L}\right\}_{n}$, we refer to a criterion originated by Aldous [1]. Following Kurtz [12] Theorem 2.7, it is sufficient to prove that for all $T>0$,

$$
\lim _{\theta \downarrow 0} \lim \sup _{n \rightarrow \infty} \sup _{\tau \in \mathscr{T}_{T}^{n}} E\left[\left|\varphi_{\tau+\theta}^{n}-\varphi_{\tau}^{n}\right|^{2}\right]=0,
$$

where $\mathscr{T}_{T}^{n}$ denotes the set of all $\left\{\mathscr{F}_{t}^{n}\right\}$-stopping times not greater than $T$. Note that $\left\{\varphi^{n}\right\}_{n}$ which we are now considering is uniformly bounded. To show (3.3), we prepare the following lemma.

Lemma 3.3. There exist a positive constant $C$, stochatic processes $M_{s, t}^{n}$, and nondecreasing processes $R_{t}^{n}$ satisfying the following (i) $\sim$ (iii):

(i) For all $s \leq t \leq T$ and $n \in \mathbf{N}$,

$$
\left|\varphi_{t}^{n}-\varphi_{s}^{n}\right|^{2} \leq C\left\{D_{t}^{n}-D_{s}^{n}+E\left[D_{t}^{n}-D_{s}^{n}\right]+\bar{D}_{t}^{n}-\bar{D}_{s}^{n}\right\}+M_{s, t}^{n}+R_{t}^{n} .
$$

(ii) For all $\tau \in \mathscr{T}_{T}^{n}, \theta>0$ and $n \in \mathbf{N}, E\left[M_{\tau, \tau+\theta}^{n}\right]=0$ holds.

(iii) For all $t, \lim _{n \rightarrow \infty} E\left[R_{t}^{n}\right]=0$ holds.

Proof. Note that $f_{u}^{n}+\bar{g}_{u}^{n}=\tilde{f}_{u}^{n}+\left(\bar{f}_{u}^{n}+\bar{g}_{u}^{n}\right)$. By integration by parts, we have

$$
\begin{aligned}
\left|\varphi_{t}^{n}-\varphi_{s}^{n}\right|^{2}= & 2 \int_{(s, t]}\left(\varphi_{u-}^{n}-\varphi_{s}^{n}\right) d \varphi_{u}^{n}+\sum_{s<u \leq t}\left|\Delta \varphi_{u}^{n}\right|^{2} \\
= & 2 \int_{(s, t]}\left(\varphi_{u-}^{n}-\varphi_{s}^{n}\right) \tilde{f}_{u}^{n}\left(\varphi_{u-}^{n}\right) d A_{u}^{n} \\
& +2 \int_{(s, t]}\left(\varphi_{u-}^{n}-\varphi_{s}^{n}\right)\left\{\bar{f}_{u}^{n}\left(\varphi_{u-}^{n}+\bar{g}_{u}^{n}\left(\varphi_{u-}^{n}\right)\right\} d A_{u}^{n}+\sum_{s<u \leq t}\left|\Delta \varphi_{u}^{n}\right|^{2}\right. \\
= & : 2 I_{1}^{n}+2 I_{2}^{n}+I_{3}^{n} .
\end{aligned}
$$

We first estimate $I_{i}^{n}$ for $i=2,3$. For $I_{2}^{n}$, we have

$$
\left|I_{2}^{n}\right| \leq 2 C_{M, L, T} \int_{(s, t]} \sup _{x \in K}\left|\bar{f}_{u}^{n}(x)+\bar{g}_{u}^{n}(x)\right| d A_{u}^{n} \leq 2 C_{M, L, T}\left(\bar{D}_{t}^{n}-\bar{D}_{s}^{n}\right),
$$

because $\left|\varphi_{u}^{n}\right| \leq C_{M, L, T}$ for all $n, u$, and $\omega$.

For $I_{3}^{n}$, since $\Delta \varphi_{u}^{n}=\left\{\tilde{f}_{u}^{n}\left(\varphi_{u^{-}}^{n}\right)+\bar{f}_{u}^{n}\left(\varphi_{u_{-}}^{n}\right)+\bar{g}_{u}^{n}\left(\varphi_{u^{-}}^{n}\right)\right\} \Delta A_{u}^{n}$ we obtain by (A.I)

$$
\begin{aligned}
\left|I_{3}^{n}\right| & \leq 2 \int_{(s, t]} \sup _{x \in K}\left\{\left|\tilde{f}_{u}^{n}(x)\right|^{2}+\left|\bar{f}_{u}^{n}(x)+\bar{g}_{u}^{n}(x)\right|^{2}\right\} \Delta A_{u}^{n} d A_{u}^{n} \\
& \leq 2\left\{D_{t}^{n}-D_{s}^{n}+\left(\bar{D}_{t}^{n}-\bar{D}_{s}^{n}\right) \sup _{n} \bar{D}_{t}^{n}\right\} .
\end{aligned}
$$


We next consider $I_{1}^{n}$. Applying the formula of the change of variables (Lemma 3.1) to the function $\left(x-\varphi_{s}^{n}\right) \tilde{f}_{u}^{n}(x)$, we have

(3.7) $\quad\left(\varphi_{u-}^{n}-\varphi_{s}^{n}\right) \tilde{f}_{u}^{n}\left(\varphi_{u-}^{n}\right)$

$$
=\int_{(s, u)} \int_{0}^{1}\left\{\tilde{f}_{u}^{n}\left(\varphi_{v-}^{n}+\theta \Delta \varphi_{v}^{n}\right)+\left(\varphi_{v-}^{n}+\theta \Delta \varphi_{v}^{n}-\varphi_{s}^{n}\right) \partial \tilde{f}_{u}^{n}\left(\varphi_{v-}^{n}+\theta \Delta \varphi_{v}^{n}\right)\right\} d \theta d \varphi_{v}^{n}
$$

Therefore,

$$
\begin{aligned}
I_{1}^{n}= & \int_{(s, t]} \int_{(s, u)} \int_{0}^{1}\left\{\tilde{f}_{u}^{n}\left(\varphi_{v-}^{n}+\theta \Delta \varphi_{v}^{n}\right)+\left(\varphi_{v-}^{n}+\theta \Delta \varphi_{v}^{n}-\varphi_{s}^{n}\right) \partial \tilde{f}_{u}^{n}\left(\varphi_{v-}^{n}+\theta \Delta \varphi_{v}^{n}\right)\right\} d \theta \\
& \times\left\{f_{v}^{n}\left(\varphi_{v-}^{n}\right)+\bar{g}_{v}^{n}\left(\varphi_{v-}^{n}\right)\right\} d A_{v}^{n} d A_{u .}^{n}
\end{aligned}
$$

For fixed $s$, set

(3.9) $h_{v, t}^{n}(x, z)=\int_{(v, t]} \int_{0}^{1}\left\{\tilde{f}_{u}^{n}(x+\theta z)+\left(x+\theta z-\varphi_{s}^{n}\right) \partial \tilde{f}_{u}^{n}(x+\theta z)\right\} d \theta d A_{u}^{n}$.

Then Fubini's theorem implies that

$$
I_{1}^{n}=\int_{(s, t)} h_{v, t}^{n}\left(\varphi_{v-}^{n}, \Delta \varphi_{v}^{n}\right)\left\{f_{v}^{n}\left(\varphi_{v-}^{n}\right)+\bar{g}_{v}^{n}\left(\varphi_{v-}^{n}\right)\right\} d A_{v}^{n} .
$$

Further, set

$$
\hat{h}_{v, t}^{n}(x, z)=E\left[h_{v, t}^{n}(x, z) \mid \mathscr{F}_{v}^{n}\right], \quad \check{h}_{v, t}^{n}(x, z)=h_{v, t}^{n}(x, z)-\hat{h}_{v, t}^{n}(x, z) .
$$

Then, by (3.11), we have

$$
\begin{aligned}
I_{1}^{n}= & \int_{(s, t)} \check{h}_{v, t}^{n}\left(\varphi_{v-}^{n}, \Delta \varphi_{v}^{n}\right)\left\{f_{v}^{n}\left(\varphi_{v-}^{n}\right)+\bar{g}_{v}^{n}\left(\varphi_{v-}^{n}\right)\right\} d A_{v}^{n} \\
& +\int_{(s, t)} \hat{h}_{v, t}^{n}\left(\varphi_{v-}^{n}, \Delta \varphi_{v}^{n}\right) \tilde{f}_{v}^{n}\left(\varphi_{v-}^{n}\right) d A_{v}^{n} \\
& +\int_{(s, t)} \hat{h}_{v, t}^{n}\left(\varphi_{v-}^{n}, \Delta \varphi_{v}^{n}\right)\left(\bar{f}_{v}^{n}\left(\varphi_{v-}^{n}\right)+\bar{g}_{v}^{n}\left(\varphi_{v-}^{n}\right)\right) d A_{v}^{n} \\
= & : I_{11}^{n}+I_{12}^{n}+I_{13}^{n} .
\end{aligned}
$$

Define $M_{s, t}^{n}=I_{11}^{n}$. We show that it satisfies (ii). For arbitrary $\tau \in \mathscr{T}_{T}^{n}$ and $\theta>0$, it holds that

$$
\begin{aligned}
E[ & \left.\int_{(\tau, \tau+\theta)} \check{h}_{v, \tau+\theta}^{n}\left(\varphi_{v-}^{n}, \Delta \varphi_{v}^{n}\right)\left\{f_{v}^{n}\left(\varphi_{v-}^{n}\right)+\bar{g}_{v}^{n}\left(\varphi_{v-}^{n}\right)\right\} d A_{v}^{n}\right] \\
= & E\left[\left.\int_{(0, T]} I_{\{\tau<v<\tau+\theta\}} E\left[\check{h}_{v, t+\theta}^{n}(x, z) \mid \mathscr{F}_{v}^{n}\right]\right|_{t=\tau, x=\varphi_{v-,}^{n}=\Delta \varphi_{v}^{n}}\right. \\
& \left.\times\left\{f_{v}^{n}\left(\varphi_{v-}^{n}\right)+\bar{g}_{v}^{n}\left(\varphi_{v-}^{n}\right)\right\} d A_{v}^{n}\right]=0,
\end{aligned}
$$

because $I_{\{\tau<v<\tau+\theta\}}$ is $\mathscr{F}_{v}^{n}$ measurable and $\tau$ is so on the set $I_{\{\tau<v<\tau+\theta\}}$. For $I_{12}^{n}$, we 
have

$$
\begin{aligned}
\left|I_{12}^{n}\right| \leq(1+ & \left.3 C_{M, L, T}\right) \\
& \times \sum_{|\alpha| \leq 1} \int_{(s, t)} \sup _{x, y \in K}\left|E\left[\int_{(v, t]} \partial_{y}^{\alpha} \tilde{f}_{u}^{n}(y) d A_{u}^{n} \mid \mathscr{F}_{v}^{n}\right] \| \tilde{f}_{v}^{n}(x)\right| d A_{v}^{n} .
\end{aligned}
$$

Hence, combining this with (3.5), and (3.6), it is easily seen that (A.I) implies that

$$
\left|I_{2}^{n}\right|+\left|I_{3}^{n}\right|+\left|I_{12}^{n}\right| \leq C\left\{D_{t}^{n}-D_{s}^{n}+\bar{D}_{t}^{n}-\bar{D}_{s}^{n}\right\}
$$

for some constant $C$ depending only on $M, L, T$. For $I_{13}^{n}$, by (A.I)-(2), we have

$$
\left|I_{13}^{n}\right| \leq C \sum_{|\alpha| \leq 1} \int_{(s, t]} E\left[\sup _{x \in K}\left|E\left[\int_{(v, t]} \partial_{x}^{\alpha} \tilde{f}_{u}^{n}(x) d A_{u}^{n} \mid \mathscr{F}_{v}^{n}\right]\right|\right] d \bar{D}_{v}^{n} .
$$

Define a nondecreasing process $R_{t}^{n}$ by the right hand side of (3.16). Then, it is easy to see that (A.I)-(2) and (A.III)-(1) imply (iii). Thus, we have completed the proof of Lemma 3.3 .

We continue the proof of Proposition 3.2. By Lemma 3.3, we have for all $\tau \in$ $\mathscr{T}_{T}^{n}$ and $0<\theta<1$

$$
E\left[\left|\varphi_{\tau+\theta}^{n}-\varphi_{\tau}^{n}\right|^{2}\right] \leq C\left\{E\left[D_{\tau+\theta}^{n, p}-D_{\tau}^{n, p}\right]+E\left[\bar{D}_{\tau+\theta}^{n}-\bar{D}_{\tau}^{n}\right]\right\}+E\left[R_{T+1}^{n}\right] .
$$

Since $\left\{D^{n, p}\right\}_{n}$ is $C$-tight, it is easy to see that

$$
\lim _{\theta \downarrow 0} \limsup _{n \rightarrow \infty} \sup _{\tau \in \mathscr{T}_{T}^{n}} P\left[D_{\tau+\theta}^{n, p}-D_{\tau}^{n, p}>\delta\right]=0
$$

for all $\delta>0$. Combining this with the uniform integrability of $D_{T+1}^{n, p}$, we obtain

$$
\lim _{\theta \downarrow 0} \limsup _{n \rightarrow \infty} \sup _{\tau \in \mathscr{T}_{T}^{n}} E\left[D_{\tau+\theta}^{n, p}-D_{\tau}^{n, p}\right]=0 .
$$

On the other hand, by (A.I)-(2)-(ii), the second term of the right hand side of (3.17) converges to 0 as $n \rightarrow \infty$. Further, by the property (iii) in Lemma 3.3, we see that (3.3) holds for $\left\{\varphi^{n, M, L}\right\}_{n}$.

\section{3-2. Identification of limit process}

In the previous section, we have shown that $\left\{\varphi^{n, M, L}\right\}_{n}$ is tight in $\mathbf{D}_{d}$ for each $M$ and $L$, namely, any subsequence of the laws of $\left\{\varphi^{n, M, L}\right\}_{n}$ contains weakly convergent subsequence. The aim of this subsection is to show that the limit laws are identified with the law of the unique solution of a stochastic differential equation. What we would like to show is stated as follows. 
Proposition 3.4. Let $Q$ be any weak limit of the laws of $\left\{\varphi^{n, M, L}\right\}_{n}$. Then, $Q$ is equal to the law of the solution $\varphi^{M, L}$ of the following stochastic differential equation:

$$
\begin{aligned}
\varphi_{t}^{M, L}= & x_{0}+\int_{(0, t]} \sigma_{L}\left(\varphi_{u-}^{M, L}, u\right) d B_{u}+\int_{(0, t]}\left(b_{L}+c_{L}\right)\left(\varphi_{u-}^{M, L}, u\right) d u \\
& +\int_{(0, t]} \int_{\left\{\|f\|^{*} \leq 1\right\}} f_{L}\left(\varphi_{u-}^{M, L}\right) \tilde{N}(d u d f) \\
& +\int_{(0, t]} \int_{\left\{1<\|f\|^{*} \leq M\right\}} f_{L}\left(\varphi_{u-}^{M, L}\right) N(d u d f),
\end{aligned}
$$

where $\sigma_{L}(x, u)=r_{L}(x) \sigma(x, u), b_{L}(x, u)=r_{L}(x) b(x, u), c_{L}(x, u)=r_{L}(x)^{2} c(x, u)$ $+a_{1}(x, u) \times r_{L}(x) \nabla_{x} r_{L}(x)$, and $f_{L}(x)=r_{L}(x) f(x)$.

By Jacod's theorem ([5], Theorem III. 2.26. p. 144), we know that the uniqueness of the solution of stochastic differential equation implies that of the martingale problem for the corresponding generator. Therefore, to show Proposition 3.4, it is sufficient to prove the following.

Proposition 3.5. Let $Q$ be any weak limit of $\left\{\varphi^{n, M, L}\right\}$. Define a linear operator $\mathscr{L}_{u}^{M, L}$ by

$$
\begin{aligned}
& \mathscr{L}_{u}^{M, L} F(x)=\frac{1}{2} \sum_{i, j=1}^{d} a_{L}^{i j}(x, u) \frac{\partial^{2} F}{\partial x^{i} \partial x^{j}}(x)+\sum_{i=1}^{d}\left(b_{L}^{i}(x, u)+c_{L}^{i}(x, u)\right) \frac{\partial F}{\partial x^{i}}(x) \\
& +\int_{\left\{\|f\|^{*} \leq M\right\}}\left\{F\left(x+f_{L}(x)\right)-F(x)-\sum_{i=1}^{d} f_{L}^{i}(x) I_{\left\{\|f\|^{*} \leq 1\right\}} \frac{\partial F}{\partial x^{i}}(x)\right\} \nu_{u}(d f),
\end{aligned}
$$

where $a_{L}(x, u)=\sigma_{L}(x, u) \sigma_{L}(x, u)^{*}$. Then, $Q$ satisfies for all $s \leq t$ and $F \in$ $\mathbf{C}_{b}^{\infty}\left(\mathbf{R}^{d}\right)$,

$$
E^{Q}\left[F\left(\varphi_{t}\right)-F\left(\varphi_{s}\right)-\int_{s}^{t} \mathscr{L}_{u}^{M, L} F\left(\varphi_{u}\right) d u \mid \mathscr{D}_{s}\right]=0
$$

where $\varphi$ denotes a canonical element of $\mathbf{D}_{d}$ and $\mathscr{D}_{s}$ denotes the right continuous sub- $\sigma$-field generated by $\left\{\varphi_{u} ; u \leq s\right\}$.

In the remainder of this section, we prove Proposition 3.5. We give the proof only in 1-dimensional case and we omit the superscripts and the subscripts $M, L$ as in the section 3-1. For the limit measure $Q$, set $J=\left\{t>0 ; Q\left(\Delta \varphi_{t} \neq 0\right)>0\right\}$, which is at most countable. To prove (3.20), it is sufficient to show that

$$
E^{Q}\left[\left\{F\left(\varphi_{t}\right)-F\left(\varphi_{s}\right)-\int_{s}^{t} \mathscr{L}_{u} F\left(\varphi_{u}\right) d u\right\} \Psi\left(\varphi_{u_{1}}, \varphi_{u_{2}}, \ldots, \varphi_{u_{m}}\right)\right]=0
$$


for all $s, t \in J^{c}, m \in \mathbf{N}, u_{i} \in J^{c}(i=1,2, \ldots, m)$ such that $u_{1}<u_{2}<\cdots<$ $u_{m} \leq s$, and for bounded continuous function $\Psi: \mathbf{R}^{m} \rightarrow \mathbf{R}^{1}$. In the sequel, we may assume that the law of $\varphi^{n}$ converges weakly to $Q$.

Applying Lemma 3.1 repeatedly as in the proof of Lemma 3.3, we can obtain for $s \leq t$ and $F \in \mathbf{C}_{b}^{\infty}\left(\mathbf{R}^{1}\right)$

$$
\begin{aligned}
F\left(\varphi_{t}^{n}\right)-F\left(\varphi_{s}^{n}\right)= & \int_{(s, t]} F^{\prime}\left(\varphi_{u-}^{n}\right) \tilde{f}_{u}^{n}\left(\varphi_{u-}^{n}\right) d A_{u}^{n} \\
& +\int_{(s, t]} F^{\prime}\left(\varphi_{u-}^{n}\right)\left(\bar{f}_{u}^{n}\left(\varphi_{u-}^{n}\right)+\bar{g}_{u}^{n}\left(\varphi_{u-}^{n}\right) d A_{u}^{n}\right. \\
& +\sum_{s<u \leq t}\left\{F\left(\varphi_{u}^{n}\right)-F\left(\varphi_{u-}^{n}\right)-F^{\prime}\left(\varphi_{u-}^{n}\right) \Delta \varphi_{u}^{n}\right\} \\
= & \int_{(s, t)} \int_{(v, t]}\left(F^{\prime} \tilde{f}_{u}^{n}\right)^{\prime}\left(\varphi_{v-}^{n}\right) d A_{u}^{n} \tilde{f}_{v}^{n}\left(\varphi_{v-}^{n}\right) d A_{v}^{n} \\
& +\int_{(s, t]} F^{\prime}\left(\varphi_{u-}^{n}\right)\left\{\bar{f}_{u}^{n}\left(\varphi_{u-}^{n}\right)+\bar{g}_{u}^{n}\left(\varphi_{u-}^{n}\right)\right\} d A_{u}^{n} \\
& +\sum_{s<u \leq t}\left\{F\left(\varphi_{u}^{n}\right)-F\left(\varphi_{u-}^{n}\right)-F^{\prime}\left(\varphi_{u-}^{n}\right) \Delta \varphi_{u}^{n}\right\} \\
& +\int_{(s, t)} \int_{(v, t]}\left(F^{\prime} \tilde{f}_{u}^{n}\right)^{\prime}\left(\varphi_{v-}^{n}\right) d A_{u}^{n}\left\{\bar{f}_{v}^{n}\left(\varphi_{v-}^{n}\right)+\bar{g}_{v}^{n}\left(\varphi_{v-}^{n}\right)\right\} d A_{v}^{n} \\
& +\int_{(s, t]} \sum_{s<v<u}\left\{F^{\prime} \tilde{f}_{u}^{n}\left(\varphi_{v}^{n}\right)-F^{\prime} \tilde{f}_{u}^{n}\left(\varphi_{v-}^{n}\right)\right. \\
& +\int_{(s, t]} F^{\prime}\left(\varphi_{s}^{n}\right) \tilde{f}_{u}^{n}\left(\varphi_{s}^{n}\right) d A_{u}^{n} \\
= & \sum_{i=1}^{6} I_{i}^{n}(s, t) .
\end{aligned}
$$

Set $J_{i}^{n}(s, t)=E\left[I_{i}^{n}(s, t) \mid \mathscr{F}_{s}^{n}\right], i=1, \ldots, 6$. We first show that the terms $J_{i}^{n}(s, t)(i=4,5,6)$ are neglected when $n$ tends to infinity. Since

$$
\begin{gathered}
\left|J_{4}^{n}(s, t)\right| \leq\|F\|_{2} \sum_{|\alpha| \leq 1} E\left[E\left[\int_{(s, t]} \sup _{x \in K}\left|E\left[\int_{(v, t]} \partial_{x}^{\alpha} \tilde{f}_{u}^{n}(x) d A_{u}^{n} \mid \mathscr{F}_{v}^{n}\right]\right|\right]\right. \\
\left.\times \sup _{x \in K}\left|\bar{f}_{v}^{n}(x)+\bar{g}_{v}^{n}(x)\right| d A_{v}^{n} \mid \mathscr{F}_{s}^{n}\right],
\end{gathered}
$$

(A.I) -(2) and (A.III)-(1) imply that for $i=4$

$$
\lim _{n \rightarrow \infty} E\left[\left|J_{i}^{n}(s, t)\right|\right]=0 .
$$

Similarly, (A.III) -(1) implies that (3.23) holds for $i=6$. For $i=5$, since we have 


$$
\begin{aligned}
\left|J_{5}^{n}(s, t)\right| \leq 2\|F\|_{3} \sum_{|\alpha| \leq 2} E\left[\int_{(s, t)} \sup _{x, y \in K}\left|E\left[\int_{(v, t]} \partial_{x}^{\alpha} \tilde{f}_{u}^{n}(y) d A_{u}^{n} \mid \mathscr{F}_{v}^{n}\right]\right|\right. \\
\left.\quad \times\left\{\left|\tilde{f}_{v}^{n}(x)\right|^{2}+\left|\bar{g}_{v}^{n}(x)+\bar{f}_{v}^{n}(x)\right|^{2}\right\} \Delta A_{v}^{n} d A_{v}^{n} \mid \mathscr{F}_{s}^{n}\right],
\end{aligned}
$$

(A.I) and (A.III) imply (3.23) for $i=5$.

Next, we consider $I_{1}^{n}(s, t)$. To show the next lemma is one of principal parts of the proof.

LEMMA 3.6. For $u_{i}(i=1, \ldots, m)$ and $\Psi$ given in $(3.21)$, set $\Psi_{s}^{n}=\Psi\left(\varphi_{u_{1}}^{n}\right.$, $\left.\ldots, \varphi_{u_{m}}^{n}\right)$ and $\Psi_{s}=\Psi\left(\varphi_{u_{1}}, \ldots, \varphi_{u_{m}}\right)$. Then we obtain

$$
\begin{gathered}
\lim _{n \rightarrow \infty} E\left[\int_{(s, t)} \int_{(v, t]} F^{\prime \prime}\left(\varphi_{v-}^{n}\right) \tilde{f}_{u}^{n}\left(\varphi_{v-}^{n}\right) \tilde{f}_{v}^{n}\left(\varphi_{v-}^{n}\right) d A_{u}^{n} d A_{v}^{n} \times \Psi_{s}^{n}\right] \\
=E\left[\int_{(s, t]} F^{\prime \prime}\left(\varphi_{v}\right) a_{1}\left(\varphi_{v}, v\right) d v \times \Psi_{s}\right] \\
\lim _{n \rightarrow \infty} E\left[\int_{(s, t)} \int_{(v, t]} F^{\prime}\left(\varphi_{v-}^{n}\right)\left(\tilde{f}_{u}^{n}\right)^{\prime}\left(\varphi_{v-}^{n}\right) \tilde{f}_{v}^{n}\left(\varphi_{v-}^{n}\right) d A_{u}^{n} d A_{v}^{n} \times \Psi_{s}^{n}\right] \\
=E\left[\int_{(s, t]} F^{\prime}\left(\varphi_{v}\right) c\left(\varphi_{v}, v\right) d v \times \Psi_{s}\right]
\end{gathered}
$$

Proof. We will give a proof only for (3.24) because (3.25) can be proved similarly. For $s \leq v \leq t, x$ and $n$, set

$$
g_{v, t}^{n}(x)=E\left[\int_{(v, t]} \tilde{f}_{u}^{n}(x) d A_{u}^{n} \mid \mathscr{F}_{v}^{n}\right] \tilde{f}_{v}^{n}(x) .
$$

Then, note that (A.I)-(1) and (A.II)-(3) imply that for all $s \leq t \leq t^{\prime} \leq T$ and $|\alpha| \leq 1$

$$
\int_{(s, t]} \sup _{x \in K}\left|\partial_{x}^{\alpha} g_{v, t^{\prime}}^{n}(x)\right| d A_{v}^{n} \leq D_{t}^{n}-D_{s}^{n}
$$

and

$$
\lim _{n \rightarrow \infty} E\left[\sup _{x \in K}\left|E\left[\int_{(s, t]} g_{v, t}^{n}(x) d A_{v}^{n} \mid \mathscr{F}_{s}^{n}\right]-\int_{s}^{t} a_{1}(x, v) d v\right|\right]=0 .
$$

Now, since $\varphi^{n}$ converges in law to $\varphi$ in $\mathbf{D}_{d}$, by Proposition 2 in Slomiński [15], we know that there exist a sequence of positive constants $\left\{\rho^{i}\right\}_{i}$ which con-

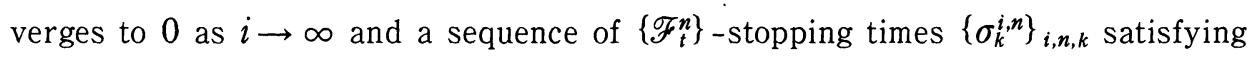
the following properties (i) $\sim$ (iii):

(i) For each $i$ and $n$

$$
0=\sigma_{0}^{i, n}<\sigma_{1}^{i, n}<\cdots, \quad \lim _{k \rightarrow \infty} \sigma_{k}^{i, n}=\infty .
$$


(ii)

$$
\lim _{i \rightarrow \infty} \limsup _{n \rightarrow \infty} P\left[\min _{k}\left\{\sigma_{k+1}^{i, n}-\sigma_{k}^{i, n} ; \sigma_{k}^{i, n} \leq T\right\} \leq \rho^{i}\right]=0
$$

(iii)

$$
\text { (3.31) } \lim _{i \rightarrow \infty} \lim \sup _{n \rightarrow \infty} P\left[\max _{k}\left\{\sup _{v \in\left[\sigma_{k}^{i,}, \sigma_{k+1}^{i, n}\right)}\left|\varphi_{v}^{n}-\varphi_{\sigma_{k}^{i, n}}^{n_{k}}\right| ; \sigma_{k}^{i, n} \leq T\right\}>\varepsilon\right]=0 \text {, }
$$

for all $\varepsilon>0$. Hence, by (3.30) and (3.31), for arbitrary $\eta, \varepsilon>0$, there exist $i_{0}$ and $n_{0}$ such that for all $i \geq i_{0}$ and $n \geq n_{0}$ we have

$$
P\left[\min _{k}\left\{\sigma_{k+1}^{i, n}-\sigma_{k}^{i, n} ; \sigma_{k}^{i, n} \leq T\right\} \leq \rho^{i}\right]<\eta,
$$

and

$$
P\left[\max _{k}\left\{\sup _{v \in\left[\sigma_{k}^{i, n}, \sigma_{k+1}^{i, n}\right)}\left|\varphi_{v}^{n}-\varphi_{\sigma_{k}^{i, n}}^{n_{i}}\right| ; \sigma_{k}^{i, n} \leq T\right\}>\varepsilon\right]<\eta .
$$

In the discussion below, we fix $\eta, \varepsilon$, and $i$ satisfying (3.32) and (3.33). For them, set

$$
\begin{aligned}
& \Omega^{n}=\left\{\omega ; \min _{k}\left\{\sigma_{k+1}^{i, n}-\sigma_{k}^{i, n} ; \sigma_{k}^{i, n} \leq T\right\}>\rho^{i}\right. \\
& \left.\quad \text { and } \max _{k}\left\{\sup _{v \in\left[\sigma_{k}^{i, n}, \sigma_{k+1}^{i, n}\right)}\left|\varphi_{v}^{n}-\varphi_{\sigma_{k}^{i_{i}, n}}^{n^{\prime}}\right| ; \sigma_{k}^{i, n} \leq T\right\} \leq \varepsilon\right\} .
\end{aligned}
$$

Set $\tau_{k}^{i, n}=\left(\sigma_{k}^{i, n} \vee s\right) \wedge t$. Then, we have

$$
\begin{aligned}
& \left|E\left[\left\{\int_{(s, t]} g_{v, t}^{n}\left(\varphi_{v-}^{n}\right) d A_{v}^{n}-\int_{(s, t]} a_{1}\left(\varphi_{v-}^{n}, v\right) d v\right\} \Psi_{s}^{n}\right]\right| \\
& \leq\left|E\left[\left\{\int_{(s, t]} g_{v, t}^{n}\left(\varphi_{v-}^{n}\right) d A_{v}^{n}-\sum_{k=0}^{T / \rho^{i}} \int_{\left(\tau_{k}^{i, n}, \tau_{k+1}^{i, n}\right.} g_{v, t}^{n}\left(\varphi_{\tau_{k}^{i, n}}^{n_{i}}\right) d A_{v}^{n}\right\} \Psi_{s}^{n}\right]\right|
\end{aligned}
$$

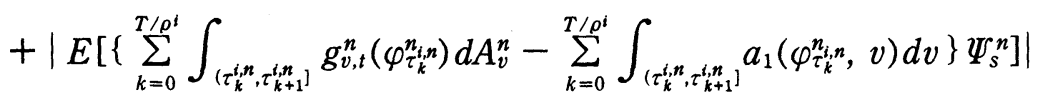

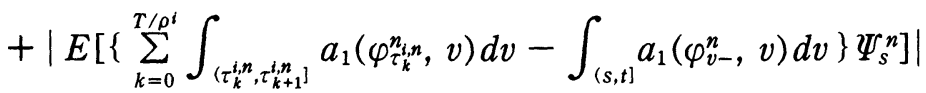

$$
\begin{aligned}
& =: J_{1}^{n}+J_{2}^{n}+J_{3}^{n} \text {. }
\end{aligned}
$$

For $J_{1}^{n},($ A.I) -(1) implies that

$$
\begin{aligned}
& J_{1}^{n} \leq \sum_{k=0}^{T / \rho^{i}} E\left[\int_{\left(\tau_{k}^{i, n}, \tau_{k+1}^{i, n}\right]}\left|g_{v, t}^{n}\left(\varphi_{v-}^{n}\right)-g_{v, t}^{n}\left(\varphi_{\tau_{k}^{t}, n}^{n}\right)\right| d A_{v}^{n}\right]\|\Psi\| \\
& \leq \sum_{k=0}^{T / \rho i} E\left[\int_{\left(\tau_{k}^{i, n}, \tau_{k+1}^{i, n}\right]} \sup _{x \in K}\left|\partial_{x} g_{v, t}^{n}(x) \| \varphi_{v-}^{n}-\varphi_{\tau_{k}^{i, n}}^{n_{i, n}}\right| d A_{v}^{n}\right]\|\Psi\| \\
& \leq \sum_{k=0}^{T / \rho i} E\left[\int_{\left(\tau_{k}^{i, n}, \tau_{k+1}^{i, n}\right]}\left|\varphi_{v-}^{n}-\varphi_{\tau_{k}^{i, n}}^{n_{i}}\right| d D_{v}^{n, p}\right]\|\Psi\|
\end{aligned}
$$




$$
\begin{aligned}
\leq & \sum_{k=0}^{T / \rho^{i}} E\left[\int_{\left(\tau_{k}^{i, n}, \tau_{k+1}^{i, n}\right)}\left|\varphi_{v-}^{n}-\varphi_{\tau_{k}^{i, n}}^{n_{i}}\right| d D_{v}^{n, p} ; \Omega^{n}\right]\|\Psi\| \\
& +\sum_{k=0}^{T / \rho^{i}} E\left[\int_{\left(\tau_{k}^{i, n}, \tau_{k+1}^{i, n}\right]}\left|\varphi_{v-}^{n}-\varphi_{\tau_{k}^{i, n}}^{n_{i, n}}\right| d D_{v}^{n, p} ;\left(\Omega^{n}\right)^{c}\right]\|\Psi\| \\
= & : J_{11}^{n}+J_{12}^{n} .
\end{aligned}
$$

Since

$$
\sup _{v \in\left(\tau_{k}^{i, n}, \tau_{k+1}^{j, n}\right]}\left|\varphi_{v-}^{n}-\varphi_{\tau_{k}^{i, n}}^{n}\right| \leq \varepsilon
$$

on $\Omega^{n}$ and $D_{t}^{n, p}$ is a nondecreasing process, we have

$$
J_{11}^{n} \leq \varepsilon \sup _{n} E\left[D_{t}^{n, p}\right]\|\Psi\| .
$$

Note that (A.I)-(1) implies that $\sup _{n} E\left[D_{t}^{n, p}\right]<\infty$ for each $t$. On the other hand, by (3.32), (3.33) and (3.34), we have

$$
\begin{aligned}
J_{12}^{n} & \leq 2 C_{M, L, T} E\left[D_{t}^{n, p} ;\left(\Omega^{n}\right)^{c}\right] \\
& \leq 2 C_{M, L, T}\left\{E\left[D_{t}^{n, p} ; D_{t}^{n, p}>1 / \sqrt{\eta}\right]+(1 / \sqrt{\eta}) P\left(\left(\Omega^{n}\right)^{c}\right)\right\} \\
& \leq 2 C_{M, L, T}\left\{E\left[D_{t}^{n, p} ; D_{t}^{n, p}>1 / \sqrt{\eta}\right]+2 \sqrt{\eta}\right\} .
\end{aligned}
$$

Combining this with (3.37), we can see that $\lim \sup _{n \rightarrow \infty} J_{1}^{n}=0$ because $\left\{D_{t}^{n, p}\right\}_{n}$ is uniformly integrable and $\eta, \varepsilon>0$ are arbitrary.

For $J_{2}^{n}$ we have

$$
\begin{aligned}
& J_{2}^{n} \leq \sum_{k=0}^{T / \rho i}\left|E\left[\left\{\int_{\left(\tau_{k}^{i, n}, t\right]} g_{v, t}^{n}\left(\varphi_{\tau_{k}^{i, n}}^{n_{i}}\right) d A_{v}^{n}-\int_{\left(\tau_{k}^{i, n}, t\right]} a_{1}\left(\varphi_{\tau_{k}}^{n_{i, n}, v}, v\right) d v\right\} \Psi_{s}^{n}\right]\right| \\
& +\sum_{k=0}^{T / \rho^{i}}\left|E\left[\left\{\int_{\left(\tau_{k+1}^{i, n}, t\right]} g_{v, t}^{n}\left(\varphi_{\tau_{k}^{i, n}}^{n_{i, n}}\right) d A_{v}^{n}-\int_{\left(\tau_{k+1}^{i, n}, t\right]} a_{1}\left(\varphi_{\tau_{k}^{t, n}}^{n_{t, n}}, v\right) d v\right\} \Psi_{s}^{n}\right]\right| \\
& =: J_{21}^{n}+J_{22}^{n} \text {. }
\end{aligned}
$$

Further, for $m \in \mathbf{N}$, set $\tau_{k}^{i, n, m}=t \wedge\left(\left[m \tau_{k}^{i, n}\right]+1\right) / m$. Then we have by (3.27)

$$
\begin{aligned}
& J_{21}^{n} \leq \sum_{k=0}^{T / \rho i}\left|E\left[\int_{\left(\tau_{k}^{i, n}, \tau_{k}^{i}, n, m_{1}\right]} g_{v, t}^{n}\left(\varphi_{\tau_{k}^{i, n}}^{n_{i}}\right) d A_{v}^{n} \Psi_{s}^{n}\right]\right| \\
& +\sum_{k=0}^{T / \rho^{i}}\left|E\left[\left\{\int_{\left(\tau_{k}^{j, n, m}, t\right]} g_{v, t}^{n}\left(\varphi_{\left.\tau_{k}^{n}, n\right)}^{n_{i}}\right) d A_{v}^{n}-\int_{\left(\tau_{k}^{j, n, m}, t\right]} a_{1}\left(\varphi_{\tau_{k}^{n}, n}^{n_{1}, v} v\right) d v\right\} \Psi_{s}^{n}\right]\right| \\
& +\sum_{k=0}^{T / \rho^{i}}\left|E\left[\int_{\left(\tau_{k}^{i, n}, \tau_{k}^{i, n, m_{1}}\right.} a_{1}\left(\varphi_{\tau_{k}^{n, n}, v}^{n_{i, n}} v\right) d A_{v}^{n} \Psi_{s}^{n}\right]\right| \\
& \leq \sum_{k=0}^{T / \rho^{\prime}} E\left[D_{\tau_{k}^{i, m}, m}^{n, p}-D_{\tau_{k}^{i, n}}^{n, p}\right]\|\Psi\|+J_{21}^{n, m}+\left\|a_{1}\right\|\|\Psi\| \frac{1}{m}\left(\frac{T}{\rho^{i}}+1\right),
\end{aligned}
$$


where we set

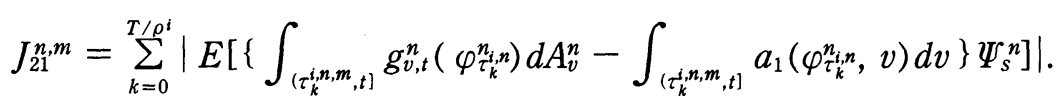

Since (A.I)-(1) implies that

$$
\lim _{m \rightarrow \infty} \limsup _{n \rightarrow \infty} \sup _{k \leq T / \rho^{i}} E\left[D_{\tau_{k}^{i, h, m}}^{n, p}-D_{\tau_{k}^{i, n}}^{n, p}\right]=0
$$

we have

$$
\lim _{m \rightarrow \infty} \limsup _{n \rightarrow \infty} \sum_{k=0}^{T / \rho^{i}} E\left[D_{\tau_{k}^{i, n, m}}^{n, p}-D_{\tau_{k}^{, i n}}^{n, p}\right]=0 .
$$

We next show that for each $m \in \mathbf{N}$

$$
\limsup _{n \rightarrow \infty} J_{21}^{n, m}=0 .
$$

To see this, note that

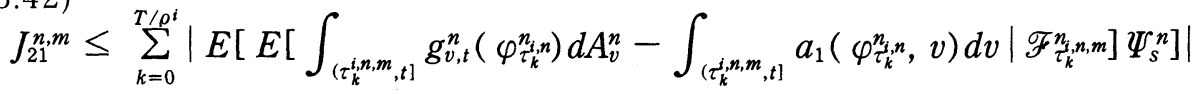

$$
\begin{aligned}
& \leq \sum_{k=0}^{T / \rho i} \sum_{l=0}^{[m t]} E\left[\sup _{x \in K} \mid E\left[\int_{(((l+1) / m) \vee s, t]} g_{v, t}^{n}(x) d A_{v}^{n}\right.\right. \\
& \left.\left.-\int_{(((l+1) / m) \vee s, t]} a_{1}(x, v) d v \mid \mathscr{F}_{((l+1) / m) \vee s}^{n}\right] \mid\right]\|\Psi\| .
\end{aligned}
$$

Therefore, by (3.28), we obtain (3.41). Combining this with (3.40), we get $\lim _{n \rightarrow \infty}$ $J_{21}^{n}=0$, because we can take $m$ as large as we want in (3.39). If we note that $\varphi_{\tau_{k}^{i n}}^{n_{i, n}}$ is $\mathscr{F}_{\tau_{k}}^{n} n$-measurable for all $k$, we can see that the similar argument on $J_{21}^{n}$ implies that $\lim _{n \rightarrow \infty} J_{22}^{n}=0$. Hence, we have shown that $\lim _{n \rightarrow \infty} J_{2}^{n}=0$.

For $J_{3}^{n}$, by the similar argument on $J_{1}^{n}$, we can show that $\lim _{n \rightarrow \infty} J_{3}^{n}=0$. Thus, we have completed the proof of Lemma 3.6.

We continue the proof of Proposition 3.5. For $I_{2}^{n}(s, t)$ and $I_{3}^{n}(s, t)$ in $(3.22)$, the following lemma holds.

Lemma 3.7. Let $\Psi_{s}^{n}$ and $\Psi_{s}$ be the functions given in Lemma 3.6. Then we have

$$
\begin{aligned}
\lim _{n \rightarrow \infty} E\left[I_{2}^{n}(s, t) \Psi_{s}^{n}\right]= & E^{Q}\left[\int_{s}^{t} F^{\prime}\left(\varphi_{u}\right) b\left(\varphi_{u}\right) d u \Psi_{s}\right] \\
& +E^{Q}\left[\int_{s}^{t} F^{\prime}\left(\varphi_{u}\right) f\left(\varphi_{u}\right) I_{\{1<\|f\| * \leq M\}} \nu_{u}(d f) d u \Psi_{s}\right]
\end{aligned}
$$

Proof. By (A.II)-(1) and (A.II)-(4), we can obtain the conclusion similarly 
as in the proof of Lemma 3.6.

Finally, we consider $I_{3}^{n}(s, t)$, for which we get the following lemma.

Lemma 3.8. Let $\Psi_{s}^{n}$ and $\Psi_{s}$ be the same functions as in Lemma 3.6. Then we have

$$
\begin{aligned}
\lim _{n \rightarrow \infty} & E\left[I_{3}^{n}(s, t) \Psi_{s}^{n}\right] \\
= & E^{Q}\left[\int_{s}^{t} a_{0}\left(\varphi_{u}, u\right) F^{\prime \prime}\left(\varphi_{u}\right) d u \Psi_{s}\right] \\
& +E^{Q}\left[\int_{s}^{t} \int_{\{\|f\| * \leq M\}}\left\{F\left(\varphi_{u}+f\left(\varphi_{u}\right)\right)-F\left(\varphi_{u}\right)-f\left(\varphi_{u}\right) F^{\prime}\left(\varphi_{u}\right)\right\}\right. \\
& \left.\times \nu_{u}(d f) d u \Psi_{s}\right]
\end{aligned}
$$

To prove this lemma, we give a preparatory lemma. Set

$$
\Phi_{u}^{n}=\int_{0}^{1} \int_{0}^{1} a F^{\prime \prime}\left(\varphi_{u-}^{n}+\alpha \beta \Delta \varphi_{u}^{n}\right) d \alpha d \beta
$$

Then, note that the mean value theorem gives

$$
I_{3}^{n}(s, t)=\sum_{s<u \leq t} \Phi_{u}^{n}\left(\Delta \varphi_{u}^{n}\right)^{2}=\int_{(s, t]} \Phi_{u}^{n}\left\{f_{u}^{n}\left(\varphi_{u-}^{n}\right)+\bar{g}_{u}^{n}\left(\varphi_{u-}^{n}\right)\right\}^{2} \Delta A_{u}^{n} d A_{u}^{n}
$$

Since it is difficult to calculate the limit of the above term directly, we interpolate it by some terms which make the limit clear as follows.

Lemma 3.9. (i) For every $\delta>0$,

(3.46) $\limsup _{n \rightarrow \infty} \mid E\left[\int_{(s, t]} \Phi_{u}^{n}\left(\left\{f_{u}^{n}\left(\varphi_{u^{-}}^{n}\right)+\bar{g}_{u}^{n}\left(\varphi_{u-}^{n}\right)\right\}^{2}\right.\right.$

$$
\left.\left.-\tilde{f}_{u, \delta}^{n}\left(\varphi_{u-}^{n}\right)^{2}-f_{u}^{n, \delta}\left(\varphi_{u-}^{n}\right)^{2}\right) \Delta A_{u}^{n} d A_{u}^{n} \Psi_{s}^{n}\right] \mid=0
$$

(ii)

(3.47) $\lim _{\delta \downarrow 0} \limsup _{n \rightarrow \infty}\left|E\left[\int_{(s, t]}\left\{\Phi_{u}^{n}-\frac{1}{2} F^{\prime \prime}\left(\varphi_{u-}^{n}\right)\right\} \tilde{f}_{u, \delta}^{n}\left(\varphi_{u-}^{n}\right)^{2} \Delta A_{u}^{n} d A_{u}^{n} \Psi_{s}^{n}\right]\right|=0$.

(iii) For every $\delta>0$,

$$
\begin{aligned}
\limsup _{n \rightarrow \infty} \mid E\left[\int _ { ( s , t ] } \left\{\Phi_{u}^{n}-\int_{0}^{1} \int_{0}^{1} \alpha F^{\prime \prime}\left(\varphi_{u-}^{n}\right.\right.\right. & \left.\left.+\alpha \beta f_{u}^{n, \delta}\left(\varphi_{u-}^{n}\right) \Delta A_{u}^{n}\right) d \alpha d \beta\right\} \\
& \left.\times f_{u}^{n, \delta}\left(\varphi_{u-}^{n}\right)^{2} \Delta A_{u}^{n} d A_{u}^{n} \Psi_{s}^{n}\right] \mid=0 .
\end{aligned}
$$


Proof. (i) Let $0<\delta \leq M$. Then $f_{u}^{n}+\bar{g}_{u}^{n}=\tilde{f}_{u, \delta}^{n}+f_{u}^{n, \delta}+\left(\bar{f}_{u, \delta}^{n}+\bar{g}_{u}^{n}\right)$ holds. Therefore

$$
\begin{aligned}
& \left(f_{u}^{n}+\bar{g}_{u}^{n}\right)^{2}-\left(\tilde{f}_{u, \delta}^{n}\right)^{2}-\left(f_{u}^{n, \delta}\right)^{2} \\
& =\left(\bar{f}_{u, \delta}^{n}+\bar{g}_{u}^{n}\right)^{2}+2 f_{u}^{n, \delta}\left(\tilde{f}_{u, \delta}^{n}+\bar{f}_{u, \delta}^{n}+\bar{g}_{u}^{n}\right)+2 \tilde{f}_{u, \delta}^{n}\left(\bar{f}_{u, \delta}^{n}+\bar{g}_{u}^{n}\right) .
\end{aligned}
$$

Since $\left|\bar{f}_{u, \delta}^{n}+\bar{g}_{u}^{n}\right| \leq \Delta \bar{D}_{u}^{n}$, we have

$$
\begin{aligned}
& \left|E\left[\int_{(s, t]} \Phi_{u}^{n}\left\{\left(f_{u}^{n}\left(\varphi_{u-}^{n}\right)+\bar{g}_{u}^{n}\left(\varphi_{u-}^{n}\right)\right)^{2}-\tilde{f}_{u, \delta}^{n}\left(\varphi_{u-}^{n}\right)^{2}-f_{u}^{n, \delta}\left(\varphi_{u-}^{n}\right)^{2}\right\} \Delta A_{u}^{n} d A_{u}^{n} \Psi_{s}^{n}\right]\right| \\
& \leq\|F\|_{2}\|\Psi\|\left\{\left(\sup _{s \leq u \leq t} \Delta \bar{D}_{u}^{n}\right)\left(\bar{D}_{t}^{n}-\bar{D}_{s}^{n}\right)\right. \\
& \quad+2 \sum_{s<u \leq t} E\left[\sup _{x \in K}\left|f_{u}^{n, \delta}(x) \Delta A_{u}^{n}\right|\right]\left(\sup _{s<u \leq t} E\left[\sup _{x \in K}\left|f_{u, \delta}^{n}(x) \Delta A_{u}^{n}\right|\right]+\sup _{s<u \leq t}\left|\Delta \bar{D}_{u}^{n}\right|\right) \\
& \left.\quad+4 \sup _{s<u \leq t} E\left[\sup _{x \in K}\left|f_{u, \delta}^{n}(x) \Delta A_{u}^{n}\right|\right]\left(\bar{D}_{t}^{n}-\bar{D}_{s}^{n}\right)\right\} .
\end{aligned}
$$

By (A.I)-(2), we have

$$
\sum_{s<u \leq t} E\left[\sup _{x \in K}\left|f_{u}^{n, \delta}(x) \Delta A_{u}^{n}\right|\right] \leq \sup _{n} \bar{D}_{t}^{n}<\infty,
$$

and $\sup _{s<u \leq t}\left|\Delta \bar{D}_{u}^{n}\right| \rightarrow 0$. Therefore the first term of the right hand side of (3.49) converges to 0 as $n \rightarrow \infty$. Further, (A.I)-(1) implies that the second term converges to 0 as $n \rightarrow \infty$. Therefore we get (3.46).

(ii) If we note that $\sup _{n} \sup _{u, x}\left|\tilde{f}_{u, \delta}^{n}(x) \Delta A_{u}^{n}\right| \leq 2\left(1+C_{M, L, T}\right) \delta$, we can see that

$$
\begin{aligned}
\mid E & {\left[\int_{(s, t]}\left\{\Phi_{u}^{n}-\frac{1}{2} F^{\prime \prime}\left(\varphi_{u-}^{n}\right)\right\} \tilde{f}_{u, \delta}^{n}\left(\varphi_{u-}^{n}\right)^{2} \Delta A_{u}^{n} d A_{u}^{n} \Psi_{s}^{n}\right] \mid } \\
\leq & \|F\|_{3}\|\Psi\|\left\{E\left[\int_{(s, t]} \sup _{x \in K}\left|\tilde{f}_{u}^{n}(x)\right| \tilde{f}_{u, \delta}^{n}(x)^{2}\left(\Delta A_{u}^{n}\right)^{2} d A_{u}^{n}\right]\right. \\
& \left.+\sup _{s<u \leq t} \sup _{x \in K}\left|\bar{g}_{u}^{n}(x)+\bar{f}_{u}^{n}(x)\right| \Delta A_{u}^{n} \times E\left[\int_{(s, t]} \sup _{x \in K} \tilde{f}_{u, \delta}^{n}(x)^{2} \Delta A_{u}^{n} d A_{u}^{n}\right]\right\} \\
\leq & \|F\|_{3}\|\Psi\|\left\{2 \delta\left(1+C_{M, L, T}\right)+\sup _{s<u \leq t} \Delta \bar{D}_{u}^{n}\right\} E\left[D_{t}^{n, p}\right] .
\end{aligned}
$$

Therefore, (A.I) implies (3.47) by the same way as in the proof of (3.46).

(iii) Note that we have

$$
\mid E\left[\int_{(s, t]}\left\{\Phi_{u}^{n}-\int_{0}^{1} \int_{0}^{1} \alpha F^{\prime \prime}\left(\varphi_{u-}^{n}+\alpha \beta f_{u}^{n, \delta}\left(\varphi_{u-}^{n}\right) \Delta A_{u}^{n}\right) d \alpha d \beta\right\} f_{u}^{n, \delta}\left(\varphi_{u-}^{n}\right)^{2}\right.
$$

$\left.\times \Delta A_{u}^{n} d A_{u}^{n} \Psi_{s}^{n}\right] \mid$ 


$$
\begin{aligned}
& \leq\|F\|_{3}\|\Psi\|\left\{E \left[\int _ { ( s , t ] } \operatorname { s u p } _ { x \in K } \left(\left|\bar{g}_{u}^{n}(x)+\bar{f}_{u, \delta}^{n}(x)\right| \Delta A_{u}^{n}\right.\right.\right. \\
& \left.\left.\quad+\left|\tilde{f}_{u, \delta}^{n}(x)\right| \Delta A_{u}^{n}\right) f_{u}^{n, \delta}(x)^{2} \Delta A_{u}^{n} d A_{u}^{n}\right] \\
& \leq\|F\|_{3}\|\Psi\|\left\{\sup _{s<u \leq t} \Delta \bar{D}_{u}^{n}+\sup _{s<u \leq t} E\left[\sup _{x \in K}\left|f_{u, \delta}^{n}(x) \Delta A_{u}^{n}\right|\right]\right\} E\left[D_{t}^{n, p}\right] .
\end{aligned}
$$

Hence, by the same argument as before, we have (3.48).

We continue the proof of Proposition 3.5. As the discussion in the proof of Lemma 3.6, we can show that (A.II)-(1) and (A.II)-(2) imply that

$$
\begin{aligned}
\lim _{\delta \downarrow 0} \limsup _{n \rightarrow \infty} \mid E\left[\int_{(s, t]} F^{\prime \prime}\left(\varphi_{u-}^{n}\right) \tilde{f}_{u, \delta}^{n}\left(\varphi_{u-}^{n}\right)^{2} \Delta A_{u}^{n} d A_{u}^{n} \Psi_{s}^{n}\right] \\
\\
-E^{Q}\left[\int_{s}^{t} F^{\prime \prime}\left(\varphi_{u}\right) a_{0}\left(\varphi_{u}\right) d u \Psi_{s}\right] \mid=0,
\end{aligned}
$$

and

(3.51) $\lim _{\delta \downarrow 0} \limsup _{n \rightarrow \infty} \mid E\left[\int_{(s, t]} \int_{0}^{1} \int_{0}^{1} \alpha F^{\prime \prime}\left(\varphi_{u-}^{n}+\alpha \beta f_{u}^{n, \delta}\left(\varphi_{u-}^{n}\right) \Delta A_{u}^{n}\right) d \alpha d \beta\right.$

$$
\left.\times f_{u}^{n, \delta}\left(\varphi_{u-}^{n}\right)^{2} \Delta A_{u}^{n} d A_{u}^{n} \Psi_{s}^{n}\right]
$$

$$
\begin{aligned}
-E^{Q} & {\left[\int_{s}^{t} \int_{\{\|f\| * \leq 1\}} \int_{0}^{1} \int_{0}^{1} \alpha F^{\prime \prime}\left(\varphi_{u}+\alpha \beta f\left(\varphi_{u}\right)\right) d \alpha d \beta f\left(\varphi_{u}\right)^{2} \nu_{u}(d f) d u \Psi_{s}\right] \mid } \\
& =0 .
\end{aligned}
$$

Since the proof is similar to that of (3.24) and (3.25), we will only consider (3.51). For any fixed $\delta>0$, set

$$
g_{u}^{n}(x)=\int_{0}^{1} \int_{0}^{1} \alpha F^{\prime \prime}\left(x+\alpha \beta f_{u}^{n, \delta}(x) \Delta A_{u}^{n}\right) d \alpha d \beta f_{u}^{n, \delta}(x)^{2} \Delta A_{u}^{n}
$$

Then, as (3.27), we have for all $s \leq t$ and $|\alpha| \leq 1$

$$
\int_{(0, t)} \sup _{x \in K}\left|\partial_{x}^{\alpha} g_{u}^{n}(x)\right| d A_{u}^{n} \ll C \times D_{t}^{n} .
$$

In fact, since $\left|f_{u}^{n, \delta}(x) \Delta A_{u}^{n}\right|=\left|f_{u, M}^{n, \delta}(x) \Delta A_{u}^{n}\right| \leq M\left(1+C_{M, L, T}\right)$, it follows from (2.4) that

$$
\begin{aligned}
& \int_{(0, t]} \sup _{x \in K}\left|\partial_{x}^{\alpha} g_{u}^{n}(x)\right| d A_{u}^{n} \\
& \ll\|F\|_{3} \sum_{u \leq t} \sup _{x \in K}\left\{\left|\partial_{x}^{\alpha} f_{u}^{n, \delta}(x) \Delta A_{u}^{n}\right|\left|f_{u}^{n, \delta}(x) \Delta A_{u}^{n}\right|^{2}+\left|\partial_{x}^{\alpha}\left(f_{u}^{n, \delta}(x) \Delta A_{u}^{n}\right)^{2}\right|\right\}
\end{aligned}
$$




$$
\begin{aligned}
& \ll\|F\|_{3} \sum_{u \leq t} \sup _{x \in K}\left\{M\left(1+C_{M, L, T}\right)\left|\partial_{x}^{\alpha} f_{u}^{n, \delta}(x) \Delta A_{u}^{n} \| f_{u}^{n, \delta}(x) \Delta A_{u}^{n}\right|\right. \\
& \left.+\left|\partial_{x}^{\alpha}\left(f_{u}^{n, \delta}(x) \Delta A_{u}^{n}\right)^{2}\right|\right\} \\
& \ll\|F\|_{3} \sum_{u \leq t} \sup _{x \in K}\left\{\left(M\left(1+C_{M, L, T}\right)+1\right)\left|\partial_{x}^{\alpha}\left(f_{u}^{n, \delta}(x) \Delta A_{u}^{n}\right)^{2}\right|\right\} \\
& \ll\left\{M\left(1+C_{M, L, T}\right)+1\right\}\|F\|_{3} D_{t}^{n} .
\end{aligned}
$$

Then we can prove (3.51) similarly as in the proof of Lemma 3.6. Thus, we have completed the proof of Proposition 3.5.

Therefore, combining Proposition 3.2 and Proposition 3.4, we obtain the following.

Proposition 3.10. For each $M \in C(\nu)$ and $L>0, \varphi^{n, M, L}$ of (3.1) converges in law to $\varphi^{M, L}$ which is the solution of (3.18).

3-3. In this subsection, we will remove the restriction of localization in the equation (3.1). Though the procedure seems routine or similar to the dicussion in section 3-5 in Fujiwara [2], we will give it for the completeness.

For each $n \in \mathbf{N}$ and $M \in C(\nu)$, we define the truncated process $\varphi^{n, M}$ of $\varphi^{n}$ by

$$
\varphi_{t}^{n, M}=x_{0}+\int_{(0, t]}\left\{f_{u, M}^{n}\left(\varphi_{u-}^{n, M}\right)+\bar{g}_{u}^{n}\left(\varphi_{u-}^{n, M}\right)\right\} d A_{u}^{n} .
$$

The aim of this subsection is to show the following proposition.

Proposition 3.11. For each $M \in C(\nu)$, the family of $\mathbf{D}_{d}$-valued random variables $\left\{\varphi^{n, M}\right\}_{n}$ converges in law to the unique solution $\varphi^{M}$ of the stochastic differential equation:

$$
\begin{aligned}
\varphi_{t}^{M}= & x_{0}+\int_{(0, t]} \sigma\left(\varphi_{u-}^{M}, u\right) d B_{u}+\int_{(0, t]}(b+c)\left(\varphi_{u-}^{M}, u\right) d u \\
& +\int_{(0, t]} \int_{\left\{\mid\|f\|^{*} \leq 1\right\}} f\left(\varphi_{u^{M}}^{M}\right) \tilde{N}(d u d f) \\
& +\int_{(0, t]} \int_{\left\{1<\|f\|^{*} \leq M\right\}} f\left(\varphi_{u-}^{M}\right) N(d u d f) .
\end{aligned}
$$

Proof. For given $L>0$, define $S_{L}(\varphi)=\inf \left\{t \geq 0 ;\left|\varphi_{t}\right| \geq L\right.$ or $\left.\left|\varphi_{t-}\right| \geq L\right\}$

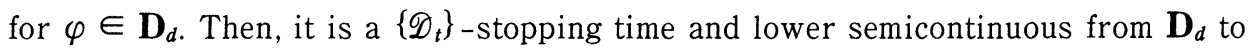
$[0, \infty]$. Set $\mathscr{D}_{S_{L}}=\left\{A \in \mathscr{D} ; A \cap\left\{S_{L} \leq t\right\} \in \mathscr{D}_{t}\right\}$ as usual. Then, since it is clear that $S_{L}\left(\varphi^{n, M, L}\right)=S_{L}\left(\varphi^{n, M}\right)$ and that $\varphi_{t}^{n, M, L}=\varphi_{t}^{n, M}$ for $t \leq S_{L}\left(\varphi^{n, M}\right)$, we have 


$$
P_{n}^{M, L}=P_{n}^{M} \quad \text { on } \quad \mathscr{D}_{S_{L}},
$$

for each $n$, where $P_{n}^{M, L}$ and $P_{n}^{M}$ denote the laws of $\varphi^{n, M, L}$ and, $\varphi^{n, M}$ respectively. Similarly, we have

$$
P^{M, L}=P^{M} \quad \text { on } \quad \mathscr{D}_{S_{L}},
$$

where $P^{M, L}$ and $P^{M}$ denote the laws of $\varphi^{M, L}$ of (3.18) and $\varphi^{M}$ of (3.54), respectively. Combining (3.55) and (3.56) with Proposition 3.10, we obtain the conclusion by a similar argument in Lemma 11.1.1 in Stroock-Varadhan [16].

3-4. In this subsection, we will remove the restriction of truncation for jumps in the equation (3.53) and complete the proof of Theorem 2.1. First, we prepare the following lemma.

Lemma 3.12. The family $\left\{\varphi^{M}\right\}_{M}$ of (3.54) converges in law to the solution of (2.16) as $M \rightarrow \infty$.

Proof. Let $N(d u d f)$ be the Poisson random measure in (2.16), and for $M>$ 0 define a stopping time $\tau_{M}$ by $\tau_{M}=\inf \left\{t ; N\left((0, t],\|f\|^{*}>M\right)>0\right\}$. Then, it is clear that $\varphi_{t}^{M}=\varphi_{t}$ if $t<\tau_{M}$, where $\varphi^{M}(\varphi)$ is the solution of (3.54) ((2.16) respectively). Hence for arbitrary $t$ and bounded $\mathscr{D}_{t}$-measurable continuous function $G$, we have

$$
\begin{aligned}
E\left[G\left(\varphi^{M}\right)\right] & \left.=E\left[G\left(\varphi^{M}\right)\right] ; t<\tau_{M}\right]+E\left[G\left(\varphi^{M}\right) ; t \geq \tau_{M}\right] \\
& \left.=E[G(\varphi)] ; t<\tau_{M}\right]+E\left[G\left(\varphi^{M}\right) ; t \geq \tau_{M}\right] \\
& =E[G(\varphi)]-E\left[G(\varphi) ; t \geq \tau_{M}\right]+E\left[G\left(\varphi^{M}\right) ; t \geq \tau_{M}\right]
\end{aligned}
$$

Therefore we have

$$
\begin{aligned}
\left|E\left[G\left(\varphi^{M}\right)\right]-E[G(\varphi)]\right| & \leq 2\|G\| P\left[\tau_{M} \leq t\right] \\
& \leq 2\|G\| P[N((0, t],\|f\| *>M)>0] \\
& =2\|G\|\left\{1-\exp \left(-\int_{0}^{t} \nu_{u}(\|f\| *>M) d u\right)\right\} \rightarrow 0
\end{aligned}
$$

as $M \rightarrow \infty$, because $\int_{0}^{t} \nu_{u}\left(\|f\|^{*}=\infty\right) d u=0$. Since $t$ is arbitrary, we can conclude that $\varphi^{M}$ converges in law to $\varphi$ of (2.16).

Finally, we show that for every bounded continuous function $G$ on $\mathbf{D}_{d}$

$$
\lim _{n \rightarrow \infty} E\left[G\left(\varphi^{n}\right)\right]=E^{Q}[G(\varphi)] .
$$


For arbitrary $t>0$, suppose that $G$ is $\mathscr{D}_{t}$-measurable. Then, it holds that

$$
\begin{gathered}
E\left[G\left(\varphi^{n}\right)\right]=E\left[G\left(\varphi^{n}\right) ; \sup _{u \leq t}\left\|f_{u}^{n} \Delta A_{u}^{n}\right\| * \leq M\right]+E\left[G\left(\varphi^{n}\right) ; \sup _{u \leq t}\left\|f_{u}^{n} \Delta A_{u}^{n}\right\|^{*}>M\right] \\
=E\left[G\left(\varphi^{n, M}\right)\right]-E\left[G\left(\varphi^{n, M}\right) ; \sup _{u \leq t}\left\|f_{u}^{n} \Delta A_{u}^{n}\right\|^{*}>M\right] \\
+E\left[G\left(\varphi^{n}\right) ; \sup _{u \leq t}\left\|f_{u}^{n} \Delta A_{u}^{n}\right\|^{*}>M\right] .
\end{gathered}
$$

Hence, we have

$$
\begin{aligned}
& \mid E\left[G\left(\varphi^{n}\right)-E^{Q}[G(\varphi)] \mid\right. \\
& \begin{aligned}
& \leq\left|E\left[G\left(\varphi^{n}\right)\right]-E\left[G\left(\varphi^{n, M}\right)\right]\right|+\mid E\left[G\left(\varphi^{n, M}\right)\right]-E\left[G\left(\varphi^{M}\right)\right] \mid \\
&+\left|E\left[G\left(\varphi^{M}\right)\right]-E^{Q}[G(\varphi)]\right| \\
& \leq 2\|G\| P\left[\sup _{u \leq t}\left\|f_{u}^{n} \Delta A_{u}^{n}\right\|^{*}>M\right]+\left|E\left[G\left(\varphi^{n, M}\right)\right]-E\left[G\left(\varphi^{M}\right)\right]\right| \\
&+
\end{aligned} \\
& +\left|E\left[G\left(\varphi^{M}\right)\right]-E^{Q}[G(\varphi)]\right| .
\end{aligned}
$$

Therefore, by (A.IV), Proposition 3.11, and Lemma 3.12, we get (3.57). Further, since $t>0$ is arbitrary, (3.57) holds for every bounded continuous function $G$.

By the above discussion, we have proved Theorem 2.1.

\section{Case of diffusions}

As applications of Theorem 2.1, we will consider a sequence of stochastic ordinary differential equations:

$$
\frac{d \varphi_{t}}{d t}=f_{t}^{n}\left(\varphi_{t}\right)
$$

where $\left\{f_{t}^{n}\right\}$ is a sequence of $\left\{\mathscr{F}_{t}^{n}\right\}$-adapted càdlàg process with values in $\mathbf{C}_{b *}^{2}$. It is assumed that $\bar{f}_{t}^{n}(x)=E\left[f_{t}^{n}(x)\right]$ exists for all $t, x$ and $\bar{f}_{t}^{n}=\bar{f}_{t}^{n}(x)$ is a

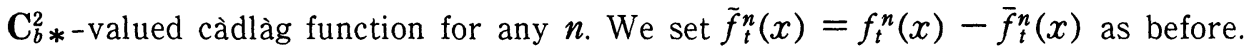
We denote by $\varphi_{t}^{n}$ the solution starting at a fixed point $x_{0}$ at time 0 . Since conditions for Theorem 2.1 can be simplified in this case, we state it for a reference. We will follow the notations in section 2 .

(A.I)': For every $N, T>0$, there exists a sequence of nondecreasing contimuous processes $\left\{D^{n}\right\}_{n}$ satisfying the following properties (i) and (ii).

(i) For all $s \leq t \leq t^{\prime} \leq T$, 


$$
\begin{array}{r}
\sum_{|\alpha| \leq 2,|\beta| \leq 1}\left\{\int_{s}^{t} \sup _{|x|,|y| \leq N}\left|E\left[\int_{v}^{t^{\prime}} \partial_{y}^{\alpha} \tilde{f}_{u}^{n}(y) d u \mid \mathscr{F}_{v}^{n}\right] \| \partial_{x}^{\beta} \tilde{f}_{v}^{n}(x)\right| d v\right. \\
\left.+\int_{s}^{t} \sup _{|x| \leq N}\left|\partial_{x}^{\beta} \bar{f}_{u}^{n}(x)\right| d u\right\} \leq D_{t}^{n}-D_{s}^{n} .
\end{array}
$$

(ii) $\left\{D^{n}\right\}_{n}$ is tight in the space $\mathbf{C}\left([0, \infty), \mathbf{R}^{1}\right)$, and $\left\{D_{t}^{n}\right\}_{n}$ is uniformly integrable for all $t$.

(A.II)': There exist a càdlàg function $a_{1}(t) \equiv a_{1}(x, t)$ with values in $\mathbf{C}_{b *}^{2}\left(\mathbf{R}^{d}, \mathbf{R}^{d} \otimes \mathbf{R}^{d}\right)$ and càdlàg functions $b(t) \equiv b(x, t), c(t) \equiv c(x, t)$ with values in $\mathbf{C}_{b *}^{1}\left(\mathbf{R}^{d}, \mathbf{R}^{d}\right)$ such that for all $N>0$ and $s \leq t$

$$
\lim _{n \rightarrow \infty} E\left[\sup _{|x| \leq N}\left|E\left[\int_{s}^{t} \int_{v}^{t} \tilde{f}_{u}^{n,(i)}(x) \tilde{f}_{v}^{n,(j)}(x) d u d v \mid \mathscr{F}_{s}^{n}\right]-\int_{s}^{t} a_{1}^{i j}(x, u) d u\right|\right]=0,
$$

and

$$
\begin{gathered}
\lim _{n \rightarrow \infty} E\left[\sup _{|x| \leq N} \mid E\left[\int_{s}^{t} \int_{v}^{t} \sum_{j=1}^{d}\left(\frac{\partial}{\partial x^{j}} \tilde{f}_{u}^{n,(i)}(x)\right) \tilde{f}_{v}^{n,(j)}(x) d u d v \mid \mathscr{F}_{s}^{n}\right]\right. \\
\left.-\int_{s}^{t} c^{i}(x, u) d u \mid\right]=0
\end{gathered}
$$

$$
\lim _{n \rightarrow \infty} \sup _{|x| \leq N}\left|\int_{s}^{t} \bar{f}_{u}^{n}(x) d u-\int_{s}^{t} b(x, u) d u\right|=0 .
$$

(A.III)': For all $N>0$ and $t>0$,

$$
\lim _{n \rightarrow \infty} \sup _{s \in[0, t]} \sum_{|\alpha| \leq 2} E\left[\sup _{|x| \leq N}\left|E\left[\int_{s}^{t} \partial_{x}^{\alpha} \tilde{f}_{u}^{n}(x) d u \mid \mathscr{F}_{s}^{n}\right]\right|\right]=0 .
$$

Then, by Theorem 2.1, we obtain the following diffusion approximation theorem. It can be regarded as an improvement of the previous work by Kunita [9].

Theorem 4.1. Assume (A.I)', (A.II)', and (A.III)'. Then, the family of continuous stochastic processes $\left\{\varphi^{n}\right\}_{n}$ satisfying (4.1) converges in law to the unique solution of the following stochastic differential equation:

$$
\varphi_{t}=x_{0}+\int_{0}^{t} \sigma\left(\varphi_{u}, u\right) d B_{u}+\int_{0}^{t}(b+c)\left(\varphi_{u}, u\right) d u
$$

where $\sigma(u)=\sigma(x, u)$ is a càdlàg function with values in $\mathbf{C}_{b *}^{1}\left(\mathbf{R}^{d}, \mathbf{R}^{d} \otimes \mathbf{R}^{\eta}\right)$ such that $\sigma(u) \sigma(u)^{*}=a_{1}(u)+a_{1}(u)^{*}$ and $B_{t}$ is an $r$-dimensional standard Brounian motion.

The solution $\varphi_{t}$ of (4.7) is often called a diffusion process with characteristics $\left(a_{1}(t)+a_{1}(t)^{*}, b(t)+c(t)\right)$. 
We shall apply the above theorem to the case where the driving processes $\left\{f_{t}^{n}(x)\right\}_{n}$ satisfy suitable mixing conditions. Define the filtrations $\left\{\mathscr{F}_{s, t}^{n}: s \leq u\right.$ $\leq t\}$ by

$$
\mathscr{F}_{s, t}^{n}=\sigma\left[f_{u}^{n} ; s \leq u \leq t\right]
$$

The strong mixing rate $\alpha^{n}(t)$ and the uniform mixing rate $\phi^{n}(t)$ of the family of the $\sigma$-fields $\left\{\mathscr{F}_{s, t}^{n}\right\}_{s \leq t}$ are defined by

$$
\begin{aligned}
\alpha^{n}(t) & =\sup _{s \geq 0} \sup _{A \in \mathscr{F}_{s+t, \infty}^{n}, B \in \mathscr{F}_{0, s}^{n}}|P(A \cap B)-P(A) P(B)|, \\
\phi^{n}(t) & =\sup _{s \geq 0} \sup _{A \in \mathscr{F}_{s+t, \infty}^{n}, B \in \mathscr{F}_{0, s}^{n}}|P(A \mid B)-P(A)| .
\end{aligned}
$$

We will first consider the case where a uniform mixing condition is satisfied. We introduce the following conditions.

(C.1): For all positive numbers $N, T$ and $|\beta| \leq 1$,

$$
\sup _{n} \sup _{t \leq T} \sup _{|x| \leq N}\left|\partial_{x}^{\beta} \bar{f}_{t}^{n}(x)\right|<\infty .
$$

(C.2): The sequence of the uniform mixing rates $\left\{\phi^{n}(t)\right\}$ satisfies

$$
\lim _{n \rightarrow \infty} \int_{0}^{T} \phi^{n}(s)^{1 / 2} d s=0
$$

for all $T$. Further the following family of random variables are uniformly integrable for any $N, T$ and $|\alpha| \leq 2$

$$
\left\{\left(\int_{0}^{T} \phi^{n}(s)^{1 / 2} d s\right) \sup _{|x| \leq N}\left|\partial_{x}^{\alpha} \tilde{f}_{t}^{n}(x)\right|^{2} ; \quad t \in[0, T], n=1,2, \ldots\right\} .
$$

(C.3): There exists a sequence $\left\{h_{n}\right\}$ of positive numbers converging to 0 such that the limits

$$
\begin{aligned}
b(x, t) & =\lim _{n \rightarrow \infty} \frac{1}{h_{n}} \int_{t}^{t+h_{n}} \bar{f}_{u}^{n}(x) d u \\
a^{i j}(x, t) & =\lim _{n \rightarrow \infty} \frac{1}{h_{n}} \int_{t}^{t+h_{n}} \int_{t}^{u} E\left[\tilde{f}_{u}^{n,(i)}(x) \tilde{f}_{v}^{n,(j)}(x)\right] d u d v \\
c^{i}(x, t) & =\lim _{n \rightarrow \infty} \frac{1}{h_{n}} \int_{t}^{t+h_{n}} \int_{t}^{u} E\left[\sum_{j=1}^{d} \frac{\partial \tilde{f}_{u}^{n,(i)}}{\partial x^{j}}(x) \tilde{f}_{v}^{n,(j)}(x)\right] d u d v
\end{aligned}
$$

exist uniformly on compact sets of $\mathbf{R}^{d} \times[0, \infty)$.

THEOREM 4.2. Assume that $\left\{f_{t}^{n}\right\}_{n}$ in equation (4.1) satisfies (C.1) $\sim(\mathrm{C} .3)$. Then $\left\{\varphi^{n}\right\}_{n}$ converges in law to a diffusion process with characteristics $\left(a(t)+a(t)^{*}\right.$, $b(t)+c(t))$. 
Before the proof we show a lemma. We fix $N, T$ and set

$$
\begin{aligned}
& K^{n}(v)=\sum_{|\alpha| \leq 2,|\beta| \leq 1}\left\{\sup _{|x| \leq N,|y| \leq N} \int_{v}^{T}\left|E\left[\partial_{y}^{\alpha} \tilde{f}_{u}^{n}(y) \mid \mathscr{F}_{v}^{n}\right]\right| d u\left|\partial_{x}^{\beta} \tilde{f}_{v}^{n}(x)\right|\right. \\
&\left.+\sup _{|x| \leq N}\left|\partial_{x}^{\beta} \bar{f}_{v}^{n}(x)\right|\right\} .
\end{aligned}
$$

LEMma 4.3. The family of random variables $\left\{K^{n}(v) ; n=1,2, \ldots, v \in[0, T]\right\}$ is uniformly integrable for any $T$.

Proof. By a uniform mixing inequality, we have

$$
\left|E\left[\partial_{y}^{\alpha} \tilde{f}_{u}^{n}(y) \mid \mathscr{F}_{v}^{n}\right]\right| \leq 2 \phi^{n}(u-v)^{\frac{1}{2}}\left\{E\left[\left|\partial_{y}^{\alpha} \tilde{f}_{u}^{n}(y)\right|^{2} \mid \mathscr{F}_{v}^{n}\right]^{\frac{1}{2}}+E\left[\left|\partial_{y}^{\alpha} \tilde{f}_{u}^{n}(y)\right|^{2}\right]^{\frac{1}{2}}\right\}
$$

See [9], Proof of Lemma 5.6.2. Consequently $K^{n}(v)$ is dominated by the sum of the following four terms.

$$
\begin{aligned}
& \int_{0}^{T-v} \phi^{n}(u)^{\frac{1}{2}} \sup _{|y| \leq N} E\left[\left|\partial_{y}^{\alpha} \tilde{f}_{u+v}^{n}(y)\right|^{2} \mid \mathscr{F}_{v}^{n}\right] d u, \\
& \int_{0}^{T-v} \phi^{n}(u)^{\frac{1}{2}} \sup _{|y| \leq N} E\left[\left|\partial_{x}^{\alpha} \tilde{f}_{u+v}^{n}(y)\right|^{2}\right] d u, \\
& \left(\int_{0}^{T-v} \phi^{n}(u)^{\frac{1}{2}} d u\right) \sup _{|x| \leq N}\left|\partial_{x}^{\beta} \tilde{f}_{v}^{n}(x)\right|^{2}, \\
& \sup _{|x| \leq N}\left|\partial_{x}^{\beta} \bar{f}_{v}^{n}(x)\right| .
\end{aligned}
$$

Clearly (4.17) and (4.19) are bounded by a positive constant not depending on $v$ and $\boldsymbol{n}$. Further (4.18) is uniformly integrable by (C.2). We will consider (4.16). By (C.2), there exists a positive convex increasing function $G(\lambda), \lambda>0$ such that $\lim _{\lambda \rightarrow \infty} G(\lambda) / \lambda=\infty$ and

$$
\sup _{n} \sup _{t \leq T} E\left[G\left(c_{n} \sup _{|y| \leq N}\left|\partial_{y}^{\alpha} \tilde{f}_{t}^{n}(y)\right|^{2}\right)\right] \equiv K<\infty,
$$

where $c_{n}=\int_{0}^{T} \phi^{n}(t)^{1 / 2} d t$. See Meyer [14]. By Jensen's inequality, we have

$$
\begin{aligned}
& E\left[G\left(\int_{0}^{T-v} \phi^{n}(u)^{\frac{1}{2}} \sup _{|y| \leq N} E\left[\left|\partial_{y}^{\alpha} \tilde{f}_{u+v}^{n}(y)\right|^{2} \mid \mathscr{F}_{v}^{n}\right] d u\right)\right] \\
& \quad \leq c_{n}^{-1} \int_{0}^{T} \phi^{n}(u)^{\frac{1}{2}} E\left[G\left(c_{n} \sup _{|y| \leq N} E\left[\left|\partial_{y}^{\alpha} \tilde{f}_{u+v}^{n}(y)\right|^{2} \mid \mathscr{F}_{v}^{n}\right]\right)\right] d u \\
& \quad \leq c_{n}^{-1} \int_{0}^{T} \phi^{n}(u)^{\frac{1}{2}} E\left[G\left(c_{n} E\left[\sup _{|y| \leq N}\left|\partial_{y}^{\alpha} \tilde{f}_{u+v}^{n}(y)\right|^{2} \mid \mathscr{F}_{v}^{n}\right]\right)\right] d u .
\end{aligned}
$$

By Jensen's inequality concerning conditional expectation, the above is bounded by 


$$
c_{n}^{-1} \int_{0}^{T} \phi^{n}(u)^{\frac{1}{2}} E\left[G\left(c_{n} \sup _{|y| \leq N}\left|\partial_{y}^{\alpha} \tilde{f}_{u+y}^{n}(y)\right|^{2}\right)\right] d u \leq K<\infty .
$$

Consequently, (4.16) is uniformly integrable.

Proof of Theorem 4.2. We take $D_{t}^{n}=\int_{0}^{t} K^{n}(v) d v$. Then, (A.I $)^{\prime}-(\mathrm{i})$ is obviously satisfied. Since $\left\{K^{n}(v) ; n=1,2, \ldots, v \in[0, T]\right\}$ is uniformly integrable, it is easily verified that $\left\{\int_{0}^{t} K^{n}(v) d v\right\}_{n}$ is also uniformly integrable for each $t$. We will prove that the latter sequence is tight. The property

$$
\lim _{c \rightarrow \infty} \sup _{n} P\left[\int_{0}^{t} K^{n}(v) d v>c\right]=0
$$

is obvious from the uniform integrability of $\left\{\int_{0}^{t} K^{n}(v) d v\right\}_{n}$. Let $0<\theta<T$ be a constant and $\sigma$ be a stopping time such that $\sigma+\theta \leq T$. Then we have

$$
\begin{aligned}
\int_{\sigma}^{\sigma+\theta} K^{n}(v) d v & =\int_{\sigma}^{\sigma+\theta} K^{n}(v) I_{[0, c]}\left(K^{n}(v)\right) d v+\int_{\sigma}^{\sigma+\theta} K^{n}(v) I_{(c, \infty)}\left(K^{n}(v)\right) d v \\
& \leq c \theta+\int_{0}^{T} K^{n}(v) I_{(c, \infty)}\left(K^{n}(v)\right) d v
\end{aligned}
$$

for any $c>0$. Therefore

$$
E\left[\int_{\sigma}^{\sigma+\theta} K^{n}(v) d v\right] \leq c \theta+\int_{0}^{T} E\left[K^{n}(v) ; K^{n}(v)>c\right] d v .
$$

For any $\varepsilon>0$, there exists $c>0$ such that $E\left[K^{n}(v) ; K^{n}(v)>c\right]<\varepsilon T^{-1}$ holds for all $n$ and $v$, since $\left\{K^{n}(v)\right\}_{n, v}$ is uniformly integrable. Next choose $\theta_{0}>0$ such that $c \theta_{0}<\varepsilon$. Then we have for any $|\theta|<\theta_{0}$

$$
\sup _{n} \sup _{\sigma \in \mathscr{T}_{T}} E\left[\int_{\sigma}^{\sigma+\theta} K^{n}(v) d v\right]<2 \varepsilon,
$$

where $\mathscr{T}_{T}$ is the set of all stopping times less than $T$. Therefore $\left\{\int_{0}{ }^{t} K^{n}(v) d v\right\}_{n}$ satisfies Aldous's condition. Then it is tight. Thus we have proved (A.I)'.

We will next prove (A.II)' . Set

$$
K^{n}(u, v, x)=\tilde{f}_{u}^{n,(i)}(x) \tilde{f}_{v}^{n,(j)}(x), \quad \bar{K}^{n}(u, v, x)=E\left[K^{n}(u, v, x)\right] .
$$

We can show similarly as in [9], Chapter 5 that (4.12) implies

$$
\lim _{n \rightarrow \infty} \int_{s}^{t} \int_{s}^{u} \bar{K}^{n}(u, v, x) d u d v=\int_{s}^{t} a^{i j}(x, r) d r,
$$

for any $s<t$. Hence it is sufficient to prove

$$
\lim _{n \rightarrow \infty} E\left[\sup _{|x| \leq N}\left|\int_{s}^{t} \int_{s}^{u} E\left[\tilde{K}^{n}(u, v, x) \mid \mathscr{F}_{s}^{n}\right] d u d v\right|\right]=0
$$


where $\tilde{K}^{n}=K^{n}-\bar{K}^{n}$. Let $c_{n}=\int_{0}^{T} \phi^{n}(s)^{1 / 2} d s$ as before. Since

$$
\left\{c_{n} \sup _{|x| \leq N}\left|f_{u}^{n}(x)\right|^{2} ; n=1,2, \ldots, u \in[0, T]\right\}
$$

is uniformly integrable, for any $\varepsilon>0$ there exists $a>0$ such that for all $n, u$

$$
E\left[c_{n} \sup _{|x| \leq N}\left|f_{u}^{n}(x)\right|^{2} ; c_{n} \sup _{|x| \leq N}\left|f_{u}^{n}(x)\right|^{2}>a\right]<\varepsilon .
$$

Now consider the truncated process $f_{u, a}^{n}=f_{u}^{n} I_{\left(\sup _{|x| \leq N}\left|f_{u}^{n}(x)\right|<\left(a / c_{n}\right)^{1 / 2}\right)}$. Set $\bar{f}_{u, a}^{n}$ $=E\left[f_{u, a}^{n}\right], \tilde{f}_{u, a}^{n}=f_{u, a}^{n}-\bar{f}_{u, a}^{n}$ and $K_{a}^{n}(u, v, x)=\tilde{f}_{u, a}^{n,(i)}(x) \tilde{f}_{v, a}^{n,(j)}(x)$. Define $\bar{K}_{a}^{n}$ and $\tilde{K}_{a}^{n}$ as above. Then $\tilde{K}^{n}=\left(\bar{K}_{a}^{n}-\bar{K}^{n}\right)+\left(K^{n}-K_{a}^{n}\right)+\tilde{K}_{a}^{n}$ holds. We have

$$
\begin{aligned}
K^{n}(u, v, x)-K_{a}^{n}( & u, v, x) \\
= & \tilde{f}_{u, a}^{n}(x)\left(\tilde{f}_{v}^{n}(x)-\tilde{f}_{v, a}^{n}(x)\right)+\left(\tilde{f}_{u}^{n}(x)-\tilde{f}_{u, a}^{n}(x)\right) \tilde{f}_{v, a}^{n}(x) \\
& +\left(\tilde{f}_{u}^{n}(x)-\tilde{f}_{u, a}^{n}(x)\right)\left(\tilde{f}_{v}^{n}(x)-\tilde{f}_{v, a}^{n}(x)\right) \\
= & : L_{1}^{n}+L_{2}^{n}+L_{3}^{n} .
\end{aligned}
$$

Apply (4.15) and (4.27) to each term of the right hand side. Then

$$
E\left[\int_{s}^{t} \int_{s}^{u} \sup _{|x| \leq N}\left|E\left[L_{i}^{n} \mid \mathscr{F}_{s}^{n}\right]\right| d u d v\right] \leq 4 T(C \varepsilon)^{1 / 2}, \quad i=1,2,
$$

where $C=\sup _{n, u}\left\{c_{n} E\left[\sup _{|x| \leq N}\left|f_{u}^{n}(x)\right|^{2}\right]\right\}$, and

$$
E\left[\int_{s}^{t} \int_{s}^{u} \sup _{|x| \leq N}\left|E\left[L_{3}^{n} \mid \mathscr{F}_{s}^{n}\right]\right| d u d v\right] \leq 4 T \varepsilon .
$$

Consequently we obtain

$$
\sup _{|x| \leq N}\left|\bar{K}_{a}^{n}-\bar{K}^{n}\right| \leq E\left[\sup _{|x| \leq N}\left|K^{n}-K_{a}^{n}\right|\right] \leq 4 T \varepsilon^{1 / 2}\left(2 C^{1 / 2}+\varepsilon^{1 / 2}\right) .
$$

Further by a uniform mixing inequality ([9], Lemma 5.6.3),

$$
\begin{aligned}
& \left|\int_{s}^{t} \int_{s}^{u} E\left[\tilde{K}_{a}^{n}(u, v, x) \mid \mathscr{F}_{s}^{n}\right] d u d v\right| \\
& \quad \leq 4 \int_{s}^{t} \int_{s}^{u} \phi^{n}(u-v)^{\frac{1}{2}} \phi^{n}(v-s)^{\frac{1}{2}}\left\|\tilde{f}_{u, a}^{n,(i)}(x)\right\|_{(\infty)}\left\|\tilde{f}_{v, a}^{n,(j)}(x)\right\|_{(\infty)} d u d v,
\end{aligned}
$$

where $\|X\|_{(p)}(1 \leq p \leq \infty)$ is the $L^{p}$ norm of the random variable $X$ with respect to the measure $P$. The right hand side of the above is dominated by

$$
\left(\int_{0}^{T} \phi^{n}(s)^{\frac{1}{2}} d s\right)^{2} \sup _{u}\left\|\tilde{f}_{u, a}^{n,(i)}(x)\right\|_{(\infty)} \sup _{v}\left\|\tilde{f}_{v, a}^{n,(j)}(x)\right\|_{(\infty)} .
$$

Since $\left\|\tilde{f}_{u, a}^{n,(i)}\right\|_{(\infty)} \leq 2\left(a / c_{n}\right)^{1 / 2}$, the above is bounded by $4 a c_{n}$. It converges to 0 a.s. as $n \rightarrow \infty$. This fact together with (4.28) proves that (4.26) holds. Therefore (4.3) 
in Condition (A.II) ${ }^{\prime}$ is verified. Other properties in (A.II) ${ }^{\prime}$ and (A.III) ${ }^{\prime}$ can be proved similarly.

We will apply Theorem 4.2 to two problems. The first is the approximation problem of stochastic differential equation.

Let $\nu^{n}(t)=\left(\nu_{1}^{n}(t), \ldots, \nu_{m}^{n}(t)\right), n=1,2, \ldots$ be a sequence of $\mathbf{R}^{m}$-valued stochastic processes with the uniform mixing rate $\phi^{n}(t)$. We assume the following $(\nu .1) \sim(\nu .3)$

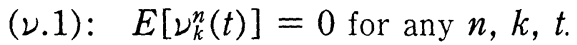

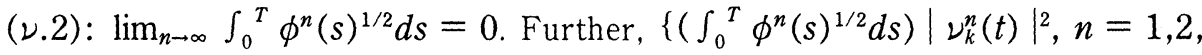
$\ldots, t \in[0, T]\}$ is uniformly integrable for any $T$.

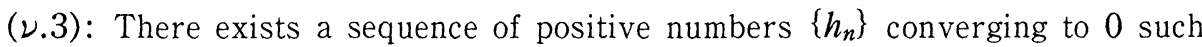
that the following limit exists uniformly on compact sets of $[0, \infty)$ :

$$
\lim _{n \rightarrow \infty} \frac{1}{h_{n}} \int_{t}^{t+h_{n}} \int_{t}^{u} E\left[\nu_{k}^{n}(u) \nu_{l}^{n}(v)\right] d u d v=v_{k l}(t) .
$$

Now define

$$
X^{n}(t)=\int_{0}^{t} \nu^{n}(s) d s
$$

It can be regarded as a solution of the equation $d \varphi_{t} / d t=\nu^{n}(t)$. Then Theorem 4.2 tells us that $\left\{X^{n}(t)\right\}$ converges in law to a Brownian $X(t)=\left(X_{1}(t), \ldots, X_{m}(t)\right)$ with mean 0 and covariance $\int_{0}^{t} v_{k l}(u) d u$.

Now let $f_{k}, k=0, \ldots, m$ be deterministic functions of $\mathbf{C}_{b *}^{2}$. Consider a sequence of stochastic ordinary differential equations:

$$
\frac{d \varphi_{t}}{d t}=f_{0}\left(\varphi_{t}, t\right)+\sum_{k=1}^{m} f_{k}\left(\varphi_{t}, t\right) \nu_{k}^{n}(t)
$$

Let $\varphi_{t}^{n}$ be the solution starting at $x_{0}$ at time 0 . Then we obtain the following from Theorem 4.2 immediately.

COROLlaRY 4.4. Assume that $\left\{\nu_{k}^{n}(t)\right\}_{n}$ sastisfies $(\nu .1) \sim(\nu .3)$. Then the sequence $\left\{\varphi^{n}\right\}_{n}$ converges in law. The limit $\varphi_{t}$ satisfies the stochastic differential equation:

$$
\varphi_{t}=x_{0}+\int_{0}^{t}\left\{f_{0}\left(\varphi_{u}, u\right)+c\left(\varphi_{u}, u\right)\right\} d u+\sum_{k=1}^{m} \int_{0}^{t} f_{k}\left(\varphi_{u}, u\right) d X_{k}(u),
$$

where $c^{i}(x, t)$ is given by

$$
c^{i}(x, t)=\sum_{j=1}^{d} \sum_{k, l=1}^{m} f_{k}^{j}(x, t) \frac{\partial}{\partial x^{j}} f_{l}^{i}(x, t) v_{k l}(t) .
$$

Now let $f(t) \equiv f(x, t)$ and $g(t) \equiv g(x, t), x \in \mathbf{R}^{d}, t \in[0, \infty)$ be $\mathbf{C}_{b *-}^{2}$ 
valued càdlàg stochastic processes. We assume that $f(t)$ is of mean 0 , i.e. $E[f(x, t)]=0$ for all $x$ and $t$. Consider a sequence of stochastic ordinary differential equations

$$
\frac{d \phi_{t}}{d t}=\frac{1}{\sqrt{n}} f\left(\phi_{t}, t\right)+\frac{1}{n} g\left(\phi_{t}, t\right)
$$

For each $n$, it has a unique global solution $\phi_{t}^{n}$ starting at any given point $x_{0}$ at time 0 . Let $n$ tend to infinity. Then $\phi_{t}^{n}$ converges to the trivial stochastic process $\psi_{t}^{\infty} \equiv x_{0}$. However if we consider the process $\varphi_{t}^{n} \equiv \psi_{n t}^{n}$, it satisfies

$$
\frac{d \varphi_{t}^{n}}{d t}=\sqrt{n} f\left(\varphi_{t}^{n}, n t\right)+g\left(\varphi_{t}^{n}, n t\right) .
$$

Then $\left\{\varphi^{n}\right\}_{n}$ does not converges to a trivial process. We shall show its weak convergence applying Theorem 4.2. A similar problem has been discussed by many authors. See e.g. Khas'minskii [7], Kesten-Papanicolaou [6], Kunita [8], [9]. The following Corollary may be considered as an improvement of Theorem 5.6.1 in [9].

COROLlary 4.5. Assume that the driving processes $f(t)=f(x, t)$ and $g(t)=$ $g(x, t)$ are stationary processes satisfying the following $(a)$ and $(b)$ :

(a): For every $N>0$ and $|\alpha| \leq 2, E\left[\sup _{|x| \leq N}\left|\partial_{x}^{\alpha} f(x, 0)\right|^{2}\right]<\infty$ and $E\left[\sup _{|x| \leq N}\left|\partial_{x}^{\alpha} g(x, 0)\right|^{2}\right]<\infty$.

(b): $\int_{0}^{\infty} \phi(s)^{1 / 2} d s<\infty$.

Then $\left\{\varphi^{n}\right\}$ converges in law to a stationary diffusion process with characteristics ( $a, b$ $+c$ ) which is given by

(4.36) $a^{i j}(x)=E\left[\left(\int_{0}^{\infty} f^{i}(x, u) d u\right) f^{j}(x, 0)\right]+E\left[\left(\int_{0}^{\infty} f^{j}(x, u) d u\right) f^{i}(x, 0)\right]$,

(4.37) $c^{i}(x)=\sum_{j=1}^{d} E\left[\left(\int_{0}^{\infty} \frac{\partial f^{i}}{\partial x^{j}}(x, u) d u\right) f^{j}(x, 0)\right]$,

(4.38) $b^{i}(x)=E\left[g^{i}(x, 0)\right]$.

Proof. Set

$$
f_{t}^{n}(x)=\sqrt{n} f(x, n t)+g(x, n t), \quad \mathscr{F}_{t}^{\dot{n}}=\mathscr{F}_{n t}, \quad \phi^{n}(t)=\phi(n t) .
$$

Then $(a)$ and $(b)$ imply (C.1) and (C.2) immediately. Further since $f(x, t)$ is stationary, we have

$$
\sqrt{n} E\left[\int_{t}^{t+1 / \sqrt{n}} \int_{t}^{u} \tilde{f}_{u}^{n,(i)}(x) \tilde{f}_{v}^{n,(j)}(x) d u d v\right]
$$




$$
\begin{aligned}
& =\frac{1}{\sqrt{n}} \int_{0}^{\sqrt{n}} E\left[\left(\int_{v}^{\sqrt{n}} f^{i}(x, u) d u\right) f^{j}(x, v)\right] d v+o(n, x, t) \\
& =E\left[\left(\int_{0}^{\infty} f^{i}(x, u) d u\right) f^{j}(x, 0)\right] \\
& \quad \quad-\frac{1}{\sqrt{n}} \int_{0}^{\sqrt{n}} E\left[\left(\int_{\sqrt{n}}^{\infty} f^{i}(x, u) d u\right) f^{j}(x, v)\right] d v+o(n, x, t) \\
& =E\left[\left(\int_{0}^{\infty} f^{i}(x, u) d u\right) f^{j}(x, 0)\right]+o(n, x, t),
\end{aligned}
$$

where $o(n, x, t)$ converges to 0 uniformly in $(x, t)$ on compact sets as $n \rightarrow \infty$. We can show similarly the existence of $b$ and $c$ in Condition (C.3).

We will next consider equation (4.1) in the case where a strong mixing condition is satisfied. We introduce a condition associated with two positive numbers $\delta, r$ :

$(\mathrm{C} .2)_{\delta, r}^{\prime}$ : The sequence of the strong mixing rates $\left\{\alpha^{n}(t)\right\}$ satisfies

$$
\lim _{n \rightarrow \infty} \int_{0}^{T} \alpha^{n}(s)^{r} d s=0
$$

for any $T$. Further, for any $N, T$ and $|\alpha| \leq 2$, the sequence

$$
\left\{\left(\int_{0}^{T} \alpha^{n}(t)^{r} d t\right) \sup _{t \leq T}\left\|\sup _{|x| \leq N}\left|\partial_{x}^{\alpha} \tilde{f}_{t}^{n}(x)\right|\right\|_{(2+\delta)}^{2}\right\}_{n}
$$

is bounded.

TheOREm 4.6. Assume Conditions (C.1), (C.2) $)_{\delta, r}$ and (C.3) for some $\delta \in$ $(0, \infty)$ and $r \in(0, \delta / 2(2+\delta)(1+d))$. Then $\left\{\varphi^{n}\right\}_{n}$ converges in law to a diffusion process with characteristics $\left(a(t)+a(t)^{*}, b(t)+c(t)\right)$.

Proof. We shall again apply Theorem 4.1. We shall prove that the sequence of the driving processes $\left\{f^{n}\right\}_{n}$ satisfies Condition (A.I)'. Condsider $K^{n}(v)$ of (4.14). It is sufficient to prove that $\left\{K^{n}(v) ; n=1,2, \ldots, v \in[0, T]\right\}$ is uniformly integrable since the fact implies the tightness and the uniform integrability of $\left\{\int_{0}^{t} K^{n}(v) d v\right\}_{n}$ as in the proof of Theorem 4.2. In the sequel we will prove

$$
\sup _{n, v} E\left[K^{n}(v)^{1+\varepsilon_{0}}\right]<\infty,
$$

for sufficiently small positive number $\varepsilon_{0}$, which implies the uniform integrability of $\left\{K^{n}(v)\right\}$ immediately.

The process $K^{n}(v)$ is bounded by the sum of the following terms. 


$$
\begin{aligned}
K_{1}^{n}(v) & =\sup _{|y| \leq N} \int_{0}^{T-v}\left|E\left[\partial_{y}^{\alpha} \tilde{f}_{u+v}^{n}(y) \mid \mathscr{F}_{v}^{n}\right]\right| d u \cdot \sup _{|x| \leq N}\left|\partial_{x}^{\beta} \tilde{f}_{v}^{n}(x)\right|, \\
K_{2}^{n}(v) & =\sup _{|x| \leq N}\left|\partial_{x}^{\beta} \bar{f}_{v}^{n}(x)\right|,
\end{aligned}
$$

where $|\alpha| \leq 2$ and $|\beta| \leq 1$. The property (4.39) is obvious for $K_{2}^{n}(v)$ by (C.1). We will prove (4.39) for $K_{1}^{n}(v)$ only. We consider the case $\alpha=\beta=0$. Since

$$
K_{1}^{n}(v) \leq \int_{0}^{T-v} \sup _{|y| \leq N}\left|E\left[\tilde{f}_{u+v}^{n}(y) \mid \mathscr{F}_{v}^{n}\right]\right| \sup _{|x| \leq N}\left|\tilde{f}_{v}^{n}(x)\right| d u,
$$

we have by Hölder's inequality,

$$
\left\|K_{1}^{n}(v)\right\|_{\left(1+\varepsilon_{0}\right)} \leq \int_{0}^{T-v}\left\|\sup _{|y| \leq N}\left|E\left[\tilde{f}_{u+v}^{n}(y) \mid \mathscr{F}_{v}^{n}\right]\right|\right\|_{\left(p\left(1+\varepsilon_{0}\right)\right)}\left\|\sup _{|x| \leq N}\left|\tilde{f}_{v}^{n}(x)\right|\right\|_{\left(q\left(1+\varepsilon_{0}\right)\right)} d u,
$$

where $p, q$ are numbers greater than 1 such that $p^{-1}+q^{-1}=1$. Next let $p^{\prime}$ and $q^{\prime}$ be positive numbers such that $\left\{p\left(1+\varepsilon_{0}\right)\right\}^{-1}=p^{\prime-1}+q^{\prime-1}$. Then by a mixing inequality ([8], Lemma 3.10.2), there exists a positive constant $C=C\left(p^{\prime}, q^{\prime}, d, N\right)$ such that

$$
\left\|\sup _{|y| \leq N}\left|E\left[\tilde{f}_{u+v}^{n}(y) \mid \mathscr{F}_{v}^{n}\right]\right|\right\|_{\left(p\left(1+\varepsilon_{0}\right)\right)} \leq C \alpha^{n}(u)^{\frac{1}{p^{\prime}(d+1)}}\left\|\sup _{|y| \leq N}\left|\tilde{f}_{u+v}^{n}(y)\right|\right\|_{\left(q^{\prime}\right)}
$$

Consequently we obtain

$$
\begin{gathered}
\left\|K_{1}^{n}(v)\right\|_{\left(1+\varepsilon_{0}\right)} \leq C\left(\int_{0}^{T} \alpha^{n}(u)^{\frac{1}{p^{\prime}(d+1)}} d u\right) \sup _{v \leq u \leq T}\left\|\sup _{|y| \leq N}\left|\tilde{f}_{u}^{n}(y)\right|\right\|_{\left(q^{\prime}\right)} \\
\times\left\|\sup _{|x| \leq N}\left|\tilde{f}_{v}^{n}(x)\right|\right\|_{\left(q\left(1+\varepsilon_{0}\right)\right) .}
\end{gathered}
$$

Set $q=(2+\delta) /\left(1+\varepsilon_{0}\right)$ and $q^{\prime}=2+\delta$. Then we have $\left(p^{\prime}(d+1)\right)^{-1}=(\delta-$ $\left.2 \varepsilon_{0}\right) /\left(1+\varepsilon_{0}\right)(2+\delta)(d+1)$. If $\varepsilon_{0}>0$ is sufficiently small, the quantity is not less than $\gamma$ of Condition $(\text { C.2 })_{\delta, r}^{\prime}$. Then (4.44) is bounded. Therefore (4.39) is satisfied for $K_{1}^{n}(v)$.

Next, property (4.3) of Condition (A.II)' can be verified similarly as in the proof of Theorem 4.2. Indeed, set $c_{n}=\int_{0}^{T} \alpha^{n}(s)^{\gamma} d s$ and define the trancated processes $f_{u, a}^{n}$ similarly as in the proof of Theorem 4.2. Then we can prove that for any $\varepsilon>0$ there exists $a>0$ such that

$$
E\left[\int_{s}^{T} \int_{s}^{u} E\left[\sup _{|x| \leq N} \mid K^{n}(u, v, x)-K_{a}^{n}(u, v, x) \| \mathscr{F}_{s}^{n}\right] d u d v\right] \leq C \varepsilon^{\delta / 2(2+\delta)},
$$

where $C$ is a constant independent of $n$ and $a$. Further, instead of the uniform mixing inequality (4.29), we have inequality:

$$
\left\|\int_{s}^{t} \int_{s}^{u} \sup _{|x| \leq N}\left|E\left[\tilde{K}_{a}^{n}(u, v, x) \mid \mathscr{F}_{s}^{n}\right]\right| d u d v\right\|_{\left(1+\varepsilon_{0}\right)}
$$




$$
\begin{gathered}
\leq C \int_{s}^{t} \int_{s}^{u} \alpha^{n}(u-v)^{r} \alpha^{n}(v-s)^{r} \sup _{|x| \leq N}\left\|\tilde{f}_{u, a}^{n,(i)}(x)\right\|_{(\infty)} \\
\quad \times \sup _{|x| \leq N}\left\|\tilde{f}_{v, a}^{n,(j)}(x)\right\|_{(\infty)} d u d v
\end{gathered}
$$

where $r=\left\{2\left(1+\varepsilon_{0}\right)(1+d)\right\}^{-1}$. See Lemma 3.10 .3 in [8]. Since $r \geq \gamma$ holds for sufficiently small $\varepsilon_{0}$, the right hand side converges to 0 as $n \rightarrow \infty$. These two facts imply (4.3).

We again consider equation (4.31) in the case where $\left\{\nu_{k}^{n}(t)\right\}_{n}$ satisfies a strong mixing condition. We introduce:

$(\nu .2)_{\delta, r}^{\prime}: \lim _{n \rightarrow \infty} \int_{0}^{T} \alpha^{n}(s)^{r} d s=0$. Further the sequence

$$
\left\{\left(\int_{0}^{T} \alpha^{n}(s)^{r} d s\right) \sup _{t \leq T}\left\|\nu_{k}^{n}(t)\right\|_{(2+\delta)}^{2}\right\}_{n}
$$

is bounded for any $T$.

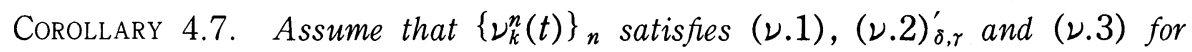
some $\delta \in(0, \infty)$ and $\gamma \in(0, \delta / 2(2+\delta))$. Then the assertion of Corollary 4.4 is valid.

Indeed inequalities (4.43) and (4.45) are valid even if we replace $p^{\prime}(d+1)$ by $p^{\prime}$ and $\left\{\left(1+\varepsilon_{0}\right)(1+d)\right\}^{-1}$ by $\left(1+\varepsilon_{0}\right)^{-1}$, respectively. See [8]. Then the corollary follows.

Finally we consider equation (4.35).

COROLlary 4.8. Assume that the driving processes $f(t)$ and $g(t)$ are stationary processes satisfying the following $(a)_{\delta}^{\prime}$ and $(b)_{r}^{\prime}$ for some $\delta \in(0, \infty)$ and $\gamma \in(0$, $\delta / 2(2+\delta)(1+d))$ :

$(a)_{\delta}^{\prime}$ : For every $N>0$ and $|\alpha| \leq 2,\left\|\sup _{|x| \leq N}\left|\partial_{x}^{\alpha} f(x, 0)\right|\right\|_{(2+\delta)}<\infty$ and $\left\|\sup _{|x| \leq N}\left|\partial_{x}^{\alpha} g(x, 0)\right|\right\|_{(2+\delta)}<\infty$.

$(b)_{\gamma}^{\prime}: \int_{0}^{\infty} \alpha(t)^{r} d t<\infty$.

Then $\left\{\varphi^{n}\right\}$ converges in law to a diffusion process with characteristics given by Corollary 4.5 .

Suppose that if $f(t)$ and $g(t)$ are represented by

$$
f(t)=\sum_{k=1}^{m} f_{k}(x) \nu_{k}(t), \quad g(t)=\sum_{k=1}^{m^{\prime}} g_{k}(x) \mu_{k}(t)+g_{0}(x),
$$


where $\left(\nu_{k}(t), \mu_{k}(t)\right)$ is a stationary process with the strong mixing rate $\alpha(t)$, then $(a)_{\delta}^{\prime}$ and $(b)_{\gamma}^{\prime}$ with $\delta \in(0, \infty)$ and $\gamma \in(0, \delta / 2(2+\delta))$ imply the weak convergence of $\left\{\varphi^{n}\right\}$.

\section{REFERENCES}

[1] D. Aldous, Stopping times and tightness, Ann. Probab., 6 (1978), 335-340.

[2] T. Fujiwara, On the jump-diffusion approximation of stochastic difference equations driven by a mixing sequence, J. Math. Soc. Japan, 42 (1990), 353-376.

[3] - Limit theorems for random difference equations driven by mixing processes, to appear in J. Math. Kyoto Univ.

[4] N. Ikeda and S. Watanabe, Stochastic Differential Equations and Diffusion Processes, North-Holland/Kodansha (1981).

[5] J. Jacod and A. N. Shiryaev, Limit Theorems for Stochastic Processes, SpringerVerlag (1987).

[6] H. Kesten and G.C. Papanicolaou, A limit theorem for turbulent diffusion, Comm. Math. Phys., 65 (1979), 97-128.

[7] R.Z. Khas'minskii, A limit theorem for the solution of differential equations with random right-hand sides, Theory Probab. Appl., 11 (1966), 390-406.

[8] H. Kunita, Stochastic Flows and Applications, Tata Institute of Fundamental Research, Springer, (1986).

[9] - Stochastic Flows and Stochastic Differential Equations, Cambridge Univ. Press, (1990).

[10] - Central limit theorems on random measures and stochastic difference equations, Gaussian Random Fields, ed. K. Itô \& T. Hida, World Scientific, (1991), $28-42$.

[11] - Limits on random measures and stochastic difference equations related to mixing array of random variables, Stochastic Analysis, ed. M.T. Barlow \& N.H. Bingham, LMS Lecture Note Series 167, Cambridge Univ. Press, (1991), 229-254.

[12] T.G. Kurtz, Approximation of Population Processes, Reginal Conference Series in Applied Mathematics 36, SIAM, (1981).

[13] H.J. Kushner, Approximation and Weak Convergence Methods for Random Proces. ses, with Applications to Stochastic System Theory, MIT Press, (1984).

[14] P.A. Meyer, Probabilités et Potentiel, Herman, (1966).

[15] L. Słomiński, Stability of strong solutions of stochastic differential equations, Stochastic Process. Appl., 31 (1989), 173-202.

[16] D.W. Stroock and S.R.S. Varadhan, Multidimensional Diffusion Processes, Springer-Verlag, (1979).

[17] H. Watanabe, Diffusion approximations of stochastic difference equations II, Hiroshima Math. J., 14 (1984), 15-34.

[18] E. Wong and M. Zakai, On the relation between ordinary and stochastic differential equations, Int. J. Eng. Sci., 3 (1965), 213-229.

Department of Applied Science

Kyushu University 36

Fukuoka 812

Japan
Current address of the first author:

Department of Mathematics

Hyogo University of Teacher Education

Yashiro, Hyogo 673-14

Japan 